\title{
Unique versus Redundant Functions of Neuroligin Genes in Shaping Excitatory and Inhibitory Synapse Properties
}

\author{
@Doham Chanda, ${ }^{1,2}$ W. Dylan Hale, ${ }^{1}{ }^{-B}$ Bo Zhang, ${ }^{1}$ Marius Wernig, ${ }^{2}$ and Thomas C. Südhof ${ }^{1}$ \\ ${ }^{1}$ Department of Molecular and Cellular Physiology and Howard Hughes Medical Institute, and ${ }^{2}$ Institute for Stem Cell Biology and Regenerative Medicine \\ and Department of Pathology, Stanford University School of Medicine, Stanford, California 94305
}

Neuroligins are evolutionarily conserved postsynaptic cell adhesion molecules that interact with presynaptic neurexins. Neurons express multiple neuroligin isoforms that are targeted to specific synapses, but their synaptic functions and mechanistic redundancy are not completely understood. Overexpression or RNAi-mediated knockdown of neuroligins, respectively, causes a dramatic increase or decrease in synapse density, whereas genetic deletions of neuroligins impair synapse function with only minor effects on synapse numbers, raising fundamental questions about the overall physiological role of neuroligins. Here, we have systematically analyzed the effects of conditional genetic deletions of all major neuroligin isoforms (i.e., NL1, NL2, and NL3), either individually or in combinations, in cultured mouse hippocampal and cortical neurons. We found that conditional genetic deletions of neuroligins caused no change or only a small change in synapses numbers, but strongly impaired synapse function. This impairment was isoform specific, suggesting that neuroligins are not functionally redundant. Sparse neuroligin deletions produced phenotypes comparable to those of global deletions, indicating that neuroligins function in a cell-autonomous manner. Mechanistically, neuroligin deletions decreased the synaptic levels of neurotransmitter receptors and had no effect on presynaptic release probabilities. Overexpression of neuroligin-1 in control or neuroligin-deficient neurons increased synaptic transmission and synapse density but not spine numbers, suggesting that these effects reflect a gain-offunction mechanism; whereas overexpression of neuroligin-3, which, like neuroligin-1 is also targeted to excitatory synapses, had no comparable effect. Our data demonstrate that neuroligins are required for the physiological organization of neurotransmitter receptors in postsynaptic specializations and suggest that they do not play a major role in synapse formation.

Key words: conditional knockout; neuroligin; primary neuronal culture; synapse development; synaptic transmission; synaptogenesis

Significance Statement

Human neuroligin genes have been associated with autism, but the cellular functions of different neuroligins and their molecular mechanisms remain incompletely understood. Here, we performed comparative analyses in cultured mouse neurons of all major neuroligin isoforms, either individually or in combinations, using conditional knockouts. We found that neuroligin deletions did not affect synapse numbers but differentially impaired excitatory or inhibitory synaptic functions in an isoform-specific manner. These impairments were due, at least in part, to a decrease in synaptic distribution of neurotransmitter receptors upon deletion of neuroligins. Conversely, the overexpression of neuroligin-1 increased synapse numbers but not spine numbers. Our results suggest that various neuroligin isoforms perform unique postsynaptic functions in organizing synapses but are not essential for synapse formation or maintenance.

\section{Introduction}

Neuroligins (NLs) are postsynaptic cell adhesion molecules that bind to presynaptic neurexins (Ichtchenko et al., 1995 and 1996;

Received Jan. 15, 2017; revised May 10, 2017; accepted May 31, 2017.

Author contributions: S.C. and T.C.S. designed research; S.C. and W.D.H. performed research; S.C., W.D.H., B.Z., and M.W. contributed unpublished reagents/analytic tools; S.C. and W.D.H. analyzed data; S.C. and T.C.S. wrote the paper.

This work was supported by grants from the National Institutes of Health (R37-MH-052804 to T.C.S.; MH-092931 to M.W.), a postdoctoral grant award (Stanford, ChEM-H112878 to S.C.), and a National Science Foundation Graduate Research Fellowship (DGE-114747, to W.D.H.).

The authors declare no competing financial interests.

Correspondence should be addressed to Soham Chanda, Department of Molecular and Cellular Physiology
Nguyen and Südhof, 1997). Mammals express four neuroligins (NL1 to NL4); of these, NL1, NL2, and NL3 are abundant and highly conserved in mice, whereas NL4 exhibits low abundance and poor conservation (Ichtchenko et al., 1996; Bolliger et al., 2008). Neuroligins form obligatory homodimers (Comoletti et al., 2003, 2006; Araç et al., 2007; Fabrichny et al., 2007; Chen et al., 2008) and may also assemble into NL1/NL3 or NL2/

and Howard Hughes Medical Institute, and Institute for Stem Cell Biology and Regenerative Medicine and Department of Pathology, Stanford University School of Medicine, Stanford, CA 94305. E-mail: schanda@stanford.edu. DOI:10.1523/JNEUROSCI.0125-17.2017

Copyright $\odot 2017$ the authors $\quad 0270-6474 / 17 / 376816-21 \$ 15.00 / 0$ 
NL3 heterodimers (Budreck and Scheiffele, 2007; Poulopoulos et al., 2012). Despite their homology and coexpression in the same neurons, neuroligins are differentially localized. NL1 is targeted to glutamatergic synapses, NL2 to GABAergic and cholinergic synapses, and NL3 to both glutamatergic and GABAergic synapses (Song et al., 1999; Varoqueaux et al., 2004; Budreck and Scheiffele, 2007; Földy et al., 2013; Takács et al., 2013).

Constitutive NL123 triple-knock-out (KO) mice displayed major impairments in synapse function but exhibited normal synapse morphology and density (Varoqueaux et al., 2006). Moreover, conditional KOs (cKOs) of neuroligins in vivo in the striatum, cerebellum, or hippocampus did not greatly alter synapse numbers (Rothwell et al., 2014; Zhang et al., 2015; Jiang et al., 2017); only $\mathrm{CKO}$ of NL2 in adult prefrontal cortex caused a modest inhibitory synapse loss that developed after 6 weeks (Liang et al., 2015). KOs of NL1 produce a major decrease in NMDA receptor (NMDAR)-mediated and, to a lesser extent, in AMPA receptor (AMPAR)-mediated synaptic responses, causing a shift in the NMDAR/AMPAR-ratio (Chubykin et al., 2007; Kim et al., 2008; Blundell et al., 2010; Soler-Llavina et al., 2011; Jedlicka et al., 2015; Jiang et al., 2017). KOs of NL2, conversely, impair synaptic transmission in subsets of GABAergic synapses (Chubykin et al., 2007; Gibson et al., 2009; Poulopoulos et al., 2009; Rothwell et al., 2014; Liang et al., 2015; Zhang et al., 2015). Overall, these data suggest that neuroligins are required for synapse maturation and function, but not for the initial formation of synaptic contacts. In some experiments using microRNA (miRNA)/short hairpin RNA (shRNA)-mediated knockdown (KD) of neuroligins, however, reduction in neuroligin expression dramatically decreased synapse and spine numbers (Chih et al., 2005; Kwon et al., 2012; Shipman et al., 2011; but for different results, see Ko et al., 2011; Soler-Llavina et al., 2011). Moreover, the overexpression of neuroligins in cultured neurons uniformly increased synapse numbers (Dean et al., 2003; Graf et al., 2004; Prange et al., 2004; Boucard et al., 2005; Chih et al., 2005; Chubykin et al., 2007; Ko et al., 2009a). Thus, in contrast to the genetic data, the RNAi and overexpression experiments appear to suggest a synaptogenic function of neuroligins.

This apparent contradiction may be explained by several differences in genetic and nongenetic approaches. Different from genetic deletions, many KD experiments were performed as sparse manipulations in which only a few neurons in a population were targeted; it is thus possible that these manipulations uncovered a phenotype that was occluded by global genetic deletions (Kwon et al., 2012). However, miRNA/shRNA-based KD experiments can produce off-target effects and broadly interfere with endogenous miRNA-based biological processes. Furthermore, rescue experiments of loss-of-function states are always overexpression experiments in which overexpressed neuroligins may exert gain-of-function effects and additionally form heterodimers with endogenous neuroligins in a nonphysiological ratio. Indeed, it remains unclear whether the increase in morphological synapses after neuroligin overexpression reflects an increased number of physiological synapses or a nonfunctional induction of synaptic specializations. Finally, multiple parallel functions of a neuroligin isoform may be occluded when one function predominates - for example, the large effect of NL1 manipulations on NMDAR-mediated responses may hinder the observation of their possible effects on AMPAR-mediated responses.

In order to address these important issues and to define the potential for functional redundancy among neuroligins, we here performed a systematic comparative analysis using acute cKOs of all major neuroligin isoforms. This approach allowed us to deconstruct the contributions of endogenous neuroligins in synaptogenesis and synapse function and to demonstrate that neuroligins perform an essential role in organizing synapses but not in the initial formation of synapses.

\section{Materials and Methods}

General experimental design. All animal experiments were performed with male and female newborn mice according to institutional guidelines and approved by the Administrative Panel on Laboratory Animal Care of Stanford University School of Medicine. All experiments except for those in Figures $1,3 F-J, 4 D$, and $11 A$ and $B$, were performed in a "blinded" fashion (i.e., the experimenter was unaware of whether a sample represented a test or control sample).

Mouse husbandry. A detailed description of generating NL cKOs (NL1 single cKO, NL2 single cKO, NL3 single cKO, NL13 double cKO, NL23 double cKO, and NL123 triple cKO) mice is described in the studies by Liang et al. (2015), Rothwell et al. (2014), and Zhang et al. (2015).

Neuronal culture. Hippocampal and cortical neurons were cultured from newborn mice as described previously (Maximov et al., 2007). Dissected hippocampi (Figs. 1, 2, 3, 4, 5, 6, 7, 8, 9, 10) or cortices (Figs. 11, 12, 13, 14) were digested for 30 min with $10 \mathrm{U} / \mathrm{ml}$ papain in HBSS buffer supplemented with $2 \mathrm{mM} \mathrm{Ca}^{2+}$ and $0.5 \mathrm{~mm}$ EGTA in an incubator, washed with HBSS buffer, dissociated in plating media (MEM supplemented with $0.5 \%$ glucose, $0.02 \% \mathrm{NaHCO} 3,0.1 \mathrm{mg} / \mathrm{ml}$ transferrin, $10 \%$ FBS, $2 \mathrm{~mm}$ L-glutamine, and $0.025 \mathrm{mg} / \mathrm{ml}$ insulin), and seeded on Matrigel (BD Biosciences) precoated coverslips placed inside 24-well dishes. The day of plating was considered as $0 \mathrm{~d}$ in vitro (DIV0). After $24 \mathrm{~h}$ (DIV1), $75 \%$ of the plating media was replaced with neuronal growth media (MEM supplemented with $0.5 \%$ glucose, $0.02 \% \mathrm{NaHCO} 3,0.1 \mathrm{mg} / \mathrm{ml}$ transferrin, 5\% FBS, 2\% B27 supplement, and $0.5 \mathrm{~mm} \mathrm{L-glutamine).} \mathrm{At}$ DIV2 (for hippocampal cultures) or DIV3 (for cortical cultures), 50\% of the medium was exchanged with fresh growth medium additionally supplemented with $4 \mu \mathrm{m}$ Ara-C (Sigma-Aldrich). The time course experiments for hippocampal and cortical synaptogenesis were performed every other day starting from DIV4 until DIV16 (Figs. 1, 11 A,B). Most of the experiments were executed at DIV14-16 (Figs. 2, 3, 4, 6, 7, 8, 9, 10, 11, $12,13)$, unless otherwise mentioned. For long-term culture of hippocampal and cortical neurons, $25 \%$ fresh media (with $4 \mu \mathrm{m}$ Ara-C) was added every $4-5 \mathrm{~d}$ starting from DIV14, and experiments were performed at DIV28 (Figs. 5, 14).

Conditional deletion of neuroligins. For global deletion of neuroligins in all neurons, we infected hippocampal or cortical neurons cultured from various single, double, or triple neuroligin cKO mice at DIV3 with lentiviruses expressing active (Cre) or inactive Cre-recombinase $(\Delta \mathrm{Cre}$; as control) that were expressed as nuclear EGFP fusion proteins under the control of human synapsin promoter (Kaeser et al., 2012). Since both Cre and $\Delta$ Cre proteins were fused to EGFP, this allowed us to directly monitor infection efficiencies. For sparse deletion of neuroligin in a small subset of neurons, we transfected the cultured neurons at DIV3 by replacing the neuronal growth media with MEM for $20 \mathrm{~min}$ and using calcium phosphate transfection with $10 \mu \mathrm{g}$ (per six coverslips) of expression vectors encoding Cre-EGFP versus $\triangle$ Cre-EGFP. The DNA/Ca ${ }^{2+}$ / HBSS mixture was added to cultured neurons for $20 \mathrm{~min}$ at $37^{\circ} \mathrm{C}$, and the neurons were washed afterward with MEM and kept in the incubator.

Neuroligin overexpression. For neuroligin overexpression experiments (Fig. 10), cultured hippocampal neurons were infected at DIV3 with lentiviruses expressing Cre-EGFP or $\Delta$ Cre-EGFP and subsequently were transfected at DIV8 with constructs encoding mVenus alone $(1 \mu \mathrm{g})$ or cotransfected with constructs encoding mVenus $(1 \mu \mathrm{g})$ and mouse NL1 (containing splice-site A and splice-site B; $1 \mu \mathrm{g}$ ) or mouse NL3 (containing splice-site A; $1 \mu \mathrm{g}$ ). All plasmids were in a pFSW67 backbone and used the human synapsin promoter; for NL1 and NL3, the endogenous signal peptides were replaced with those of Ig $\kappa$, followed by an $\mathrm{N}$-terminal HA peptide fused to the mature coding sequence.

Lentivirus production. Lentiviruses were prepared using protocols described previously (Chanda et al., 2016). In brief, human embryonic kidney 293 cells cultured in $10 \mathrm{~cm}$ dishes were cotransfected with Cre/ $\Delta$ Cre-EGFP vectors $(18 \mu \mathrm{g})$ and three helper plasmids (pRSV-REV, 
A

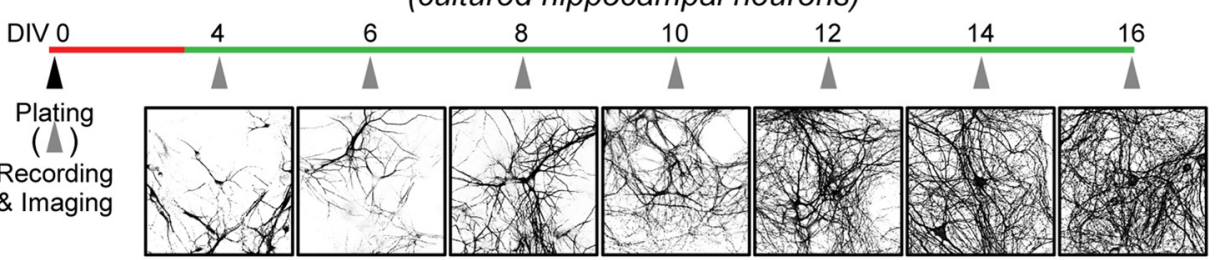

B Electrical properties

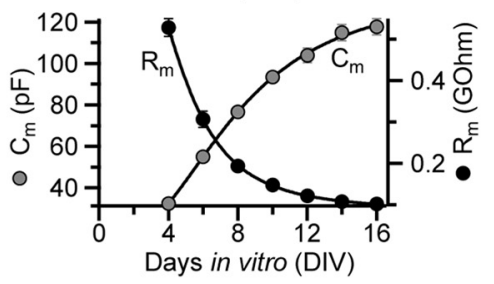

Synapse properties as a function of days in vitro

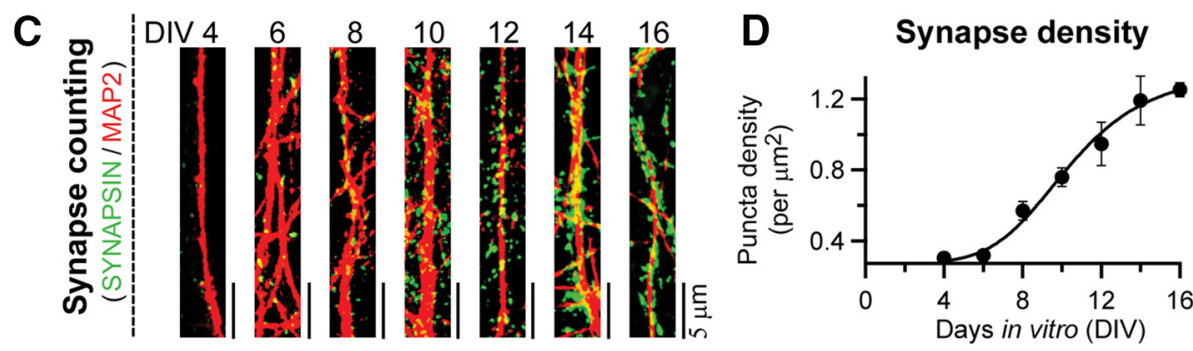

Synapse size

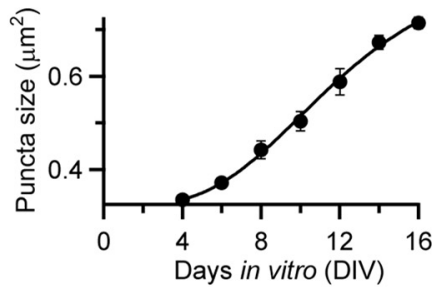

Spontaneous miniature postsynaptic currents as a function of days in vitro

E DIV 4 AMPAR mEPSCs

\begin{tabular}{l|l|l|}
\hline DIV 6 \\
\hline DIV 8 \\
\hline DIV 10
\end{tabular}

DIV 14

DIV 16

mEPSC amplitude

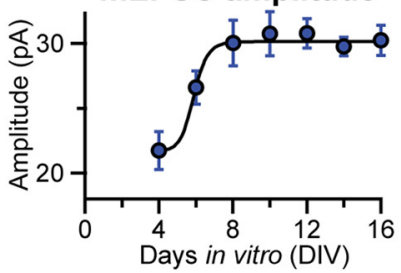

$\mathrm{2s}_{\mathrm{s}} 20 \mathrm{pA}$

mEPSC frequency

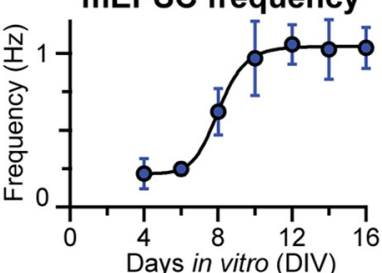

$\mathbf{F}$ DIV $4 \quad$ mIPSCs

Figure 1. Time course of excitatory and inhibitory synaptogenesis in cultured hippocampal neurons. $A$, Schematic illustration of experimental design (top) and representative images of neuronal morphology as a function of in vitro culture time (bottom, visualized by MAP2 immunostaining). Hippocampal neurons were cultured from newborn mice (black arrowhead), and electrophysiological and morphological parameters of synaptic maturation were monitored at DIV4 to DIV16 (gray arrowheads) as described in $\boldsymbol{B}-\boldsymbol{F} . \boldsymbol{B}, \boldsymbol{C}_{\mathrm{m}}$ and $\mathrm{R}_{\mathrm{m}}$ of hippocampal neurons mature gradually in culture. The capacitance and input resistance values (mean \pm SEM) are plotted as a function of culture time ( $n=51-75$ neurons/time point from three independent cultures). Data were fitted to a Hill function $\left(P=P_{\max } /\left[1+(\text { bar graph/DIV })^{n}\right]\right.$, where $P=C_{m}$ or $R_{m}$; for $C_{m}, P_{\max }=135.46 \pm 14.2 p F, n=2.54 \pm 0.73$, and $K_{1 / 2}=7.78 \pm 0.45 \mathrm{~d}$; for $R_{m}, P_{\max }=86.13 \pm 12.5 \mathrm{M} \Omega, n=3.05 \pm$ 0.82 , and $K_{1 / 2}=4.46 \pm 1.29 \mathrm{~d}$. $\boldsymbol{C}, \boldsymbol{D}, 0$ verall synaptogenesis proceeds continuously from DIV4 to DIV16 in cultured hippocampal neurons. Neurons were double labeled by immunofluorescence for MAP2 (red) and synapsin (green). The density of synaptic puncta per MAP2-positive area and the size of synaptic puncta were measured [ $\boldsymbol{C}$, representative images; $\boldsymbol{D}$, plots of synaptic puncta density and size as a function of culture time (mean \pm SEM; $n=20$ fields/DIV, 3 independent batches)]. In $\boldsymbol{D}$, data were fitted to a Hill function (see legend to $\boldsymbol{B}$ ); for synapse density, $P_{\text {max }}=1.36 \pm 0.12$ puncta/ $\mu \mathrm{m}^{2}, n=4.99 \pm 1.41$, and $\mathrm{K}_{1 / 2}=10.37 \pm 0.71 \mathrm{~d}$; for synapse size, $P_{\max }=0.87 \pm 0.13 \mu \mathrm{m}^{2}, n=3.32 \pm 0.9$, and $\mathrm{K}_{1 / 2}=12 \pm 1.82 \mathrm{~d}$. E, Measurements of spontaneous mEPSCs uncover early formation of functional glutamatergic synapses in cultured hippocampal neurons [top, representative traces; bottom, summary plots of the mEPSC amplitude (left) and frequency (right); data are means \pm SEM; $n=49-87$ cells/DIV]. Data were fitted to a Hill function (see legend for $\boldsymbol{B}$ ); for the mEPSC amplitude, $P_{\max }=30.15 \pm 0.51 \mathrm{pA}, n=15.89 \pm 104$, and $\mathrm{K}_{1 / 2}=$ $5.88 \pm 0.87 \mathrm{~d}$; for the mEPSC frequency, $P_{\max }=1.05 \pm 0.09 \mathrm{~Hz}, n=11.31 \pm 10.6$, and $\mathrm{K}_{1 / 2}=8.04 \pm 0.57 \mathrm{~d}$. $\boldsymbol{F}$, Same as $\boldsymbol{E}$, except for GABA $\mathrm{R}$-mediated mIPS(S, $n=64-114$ cells/DIV). For mIPSC amplitude, $P_{\max }=85.99 \pm 11.4 \mathrm{pA}, n=3.67 \pm 2.04$, and $\mathrm{K}_{1 / 2}=9.62 \pm 1.38 \mathrm{~d}$; and for mIPSC frequency, $P_{\max }=3.02 \pm 0.6 \mathrm{~Hz}, n=5.62 \pm 0.95$, and $\mathrm{K}_{1 / 2}=12.46 \pm 1.11 \mathrm{~d}$.

pMDLg/pRRE, and VSV-G, $9 \mu \mathrm{g}$ each) using calcium phosphate transfection. After a complete media exchange at $12 \mathrm{~h}$, supernatants were subsequently collected at 36 and $60 \mathrm{~h}$. Each $20 \mathrm{ml}$ of the pooled supernatants was centrifuged at 23,000 rpm for $2 \mathrm{~h}$, and the viral pellet was resuspended in $100 \mu \mathrm{l}$ of HBSS. The viruses were stored at $-80^{\circ} \mathrm{C}$ for no more than 2 weeks before use (1-2 $\mu \mathrm{l} /$ coverslip).

Quantitative RT-PCR. Hippocampal cultures of NL1 single cKO, NL2 single cKO, NL3 single cKO, NL13 double cKO, NL23 double cKO and
NL123 triple cKO animals were globally infected with lentivirus expressing Cre vs $\Delta$ Cre -EGFP at DIV3, and total mRNA was collected at DIV14-16, using RNeasy mini kit (Qiagen). A total of $200 \mathrm{ng}$ of total mRNA from each experimental condition was reverse transcribed into cDNA using the First Strand cDNA Synthesis Kit for RT-PCR (Life Technologies). Quantitative RT-PCR was performed using the AB7900HT Real-Time PCR system (Life Technologies) and TaqMan Gene Expression Assays (Life Technologies) for NL1 (Mm01291458_m1), NL2 
A
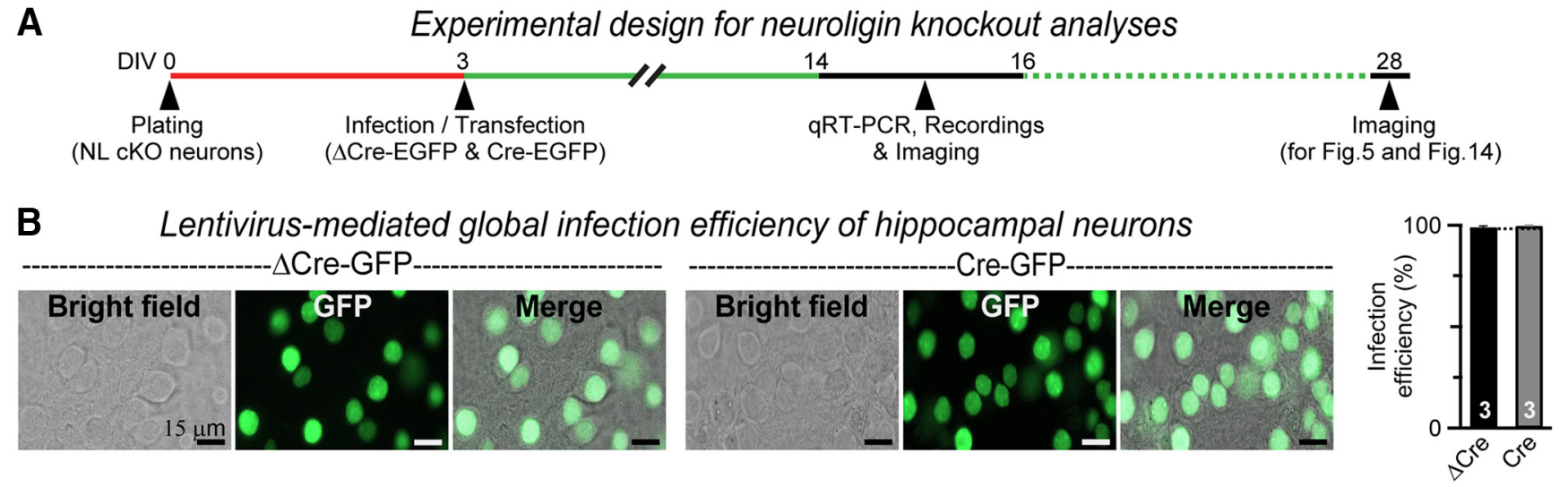

Neuroligin mRNA and protein measurements from hippocampal neurons

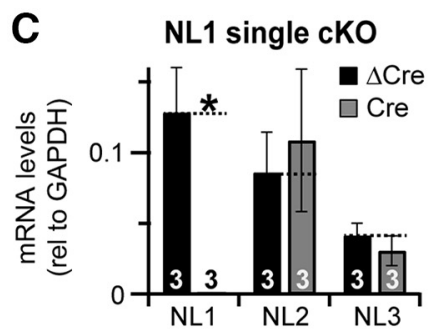

NL13 double cKO

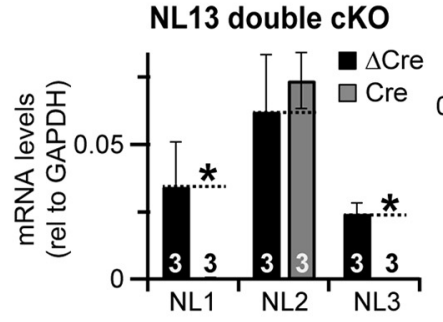

NL2 single cKO

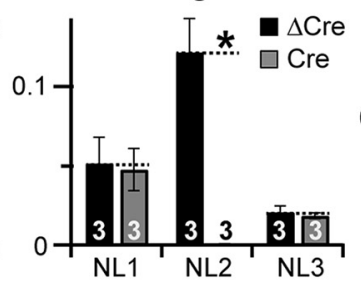

NL23 double cKO

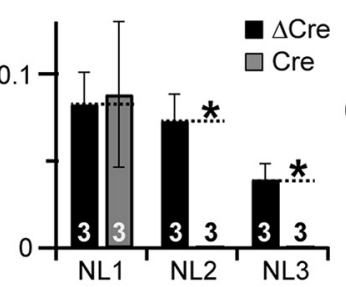

NL3 single cKO

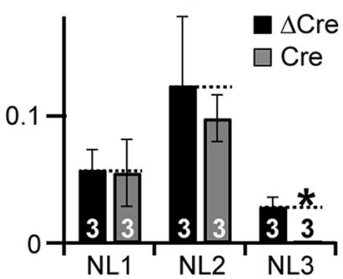

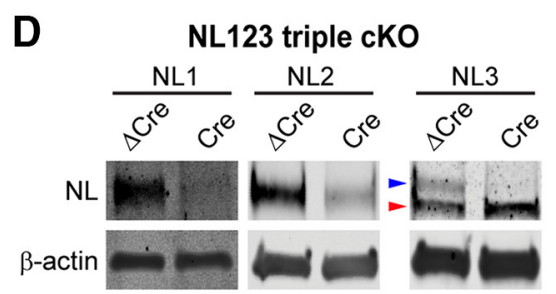

E

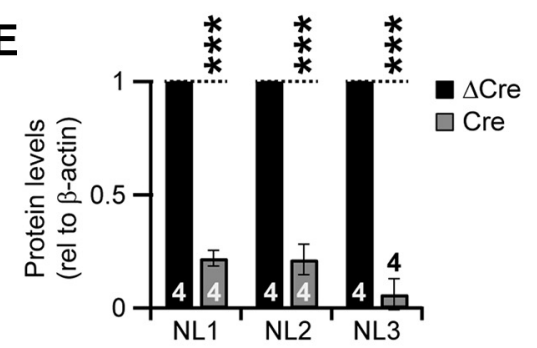

$\mathrm{NL} 1 \mathrm{NL2}$ NL3
$\mathbf{F}$

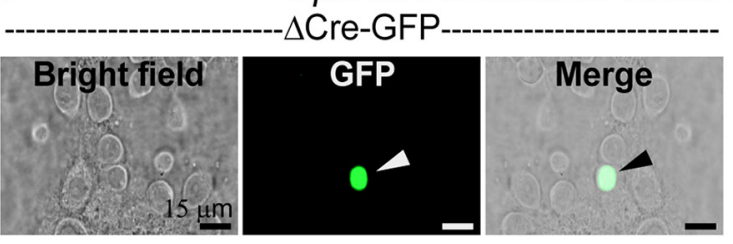

NL123 triple cKO

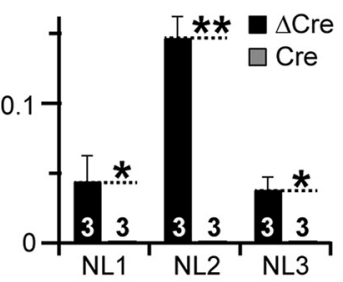

Figure 2. Validation of acute $\mathrm{CKO}$ of neuroligins in cultured hippocampal neurons using lentiviral expression of Cre-recombinase in all neurons or transfection of Cre-recombinase in a subset of neurons, with $\Delta$ Cre as a negative control in all cases. $A$, Strategy for neuroligin cK0 analysis. Hippocampal neurons cultured from newborn neuroligin cK0 mice were uniformly infected at DIV 3 with lentiviruses expressing EGFP-tagged wild-type Cre-recombinase (Cre) or a mutant version of the same ( $\Delta$ Cre), or alternatively sparsely transfected at DIV 3 with plasmids encoding the same proteins. All subsequent analyses of neuroligin K0s were performed at DIV14-16, if not mentioned otherwise. Particularly for Figure 5 and Fig. 14, DIV3-infected Cre- or $\Delta$ Cre-expressing neurons were cultured for an extended period and were analyzed at DIV28. B, Lentiviral infection expresses Cre or $\Delta$ Cre in all neurons (left, representative images; right, summary graphs of the infection efficiency quantified via the nuclear EGFP signal of lentivirally encoded (re-EGFP or $\Delta$ (re-EGFP). C, Quantitative RT-PCR measurements confirm that lentiviral Cre expression at DIV3 reduces neuroligin expression below the detection limit at DIV14-16. Bar graphs show GAPDH-normalized neuroligin mRNA expressions for NL1, NL2, and NL3, in cultured hippocampal neurons expressing $\Delta$ Cre (black) or Cre (gray); neurons were cultured from the indicated cK0 mice. $\boldsymbol{D}, \boldsymbol{E}$, Representative images (D) and summary graphs $(\boldsymbol{E})$ of Western blot analysis for NL1, NL2, and NL3 protein expressions in DIV14-16 primary hippocampal neurons derived from NL123 triple CKO animals, which were infected with lentivirus encoding either $\Delta$ Cre or Cre at DIV3. The neuroligin signals (NL) were normalized to corresponding $\beta$-actin signals for quantifications in $\boldsymbol{E}$. Arrowheads in $\boldsymbol{D}$ indicate NL3 signal (blue) and a nonspecific band (red). $\boldsymbol{F}$, Same as $\boldsymbol{B}$, except for $\mathrm{Ca}^{2+}$ phosphate-mediated sparse transfection of cultured hippocampal neurons. For all summary graphs, average values are presented as the mean \pm SEM. along with numbers of independent cultures. Asterisks show significant differences between $\Delta$ Cre and Cre conditions, as assessed by paired, one-tailed $t$ test $\left({ }^{*} p<0.05 ;{ }^{* *} p<0.01 ;{ }^{* * *} p<0.005\right)$.

(Mm01245478_m1), and NL3 (Mm01225951_m1). Average cycle threshold $\left(\mathrm{C}_{\mathrm{t}}\right)$ values for the mRNA levels of each neuroligin isoforms were calculated for all genotypes and were normalized to that of GAPDH (Mm99999915_g1).

Immunoblotting experiments. DIV14-16 primary hippocampal cultures derived from NL123 triple cKO animals were lysed with RIPA buffer (50 mм Tris, $250 \mu \mathrm{M} \mathrm{NaCl}, 1 \%$ Triton X-100, 1\% sodium deoxycholate, and $0.2 \%$ SDS, pH 7.5) with fresh protease inhibitors (Sigma-
Aldrich). Crude lysates were collected and boiled in sample buffer. Total extracted protein $(20-30 \mu \mathrm{g})$ was loaded onto a $4-20 \%$ Tris-glycine gradient gel (Bio-Rad) before being transferred to a nitrocellulose membrane. Membranes were immunostained overnight with the following primary antibodies: mouse NL1 (1:1000; Synaptic Systems; RRID: AB_887747); mouse NL2 (1:500; Synaptic Systems; RRID: AB_2619813); or rabbit NL3 (1:500; 639B, Südhof laboratory). Blots were stained with fluorescent secondary antibodies (LI-COR) and were imaged on a 
NL123 triple cKO - global cKO in lentivirally infected neurons

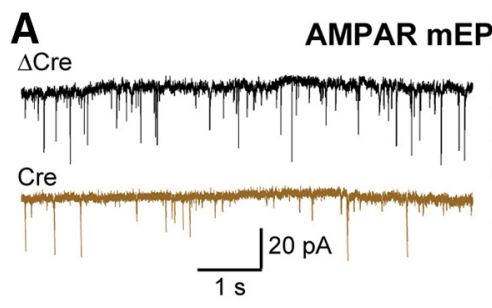

C
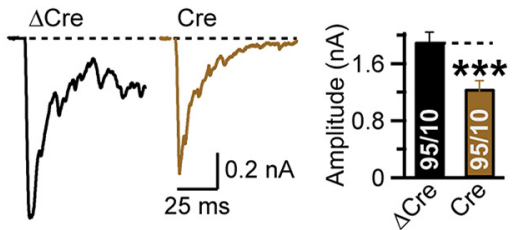

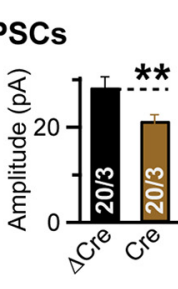

D

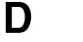

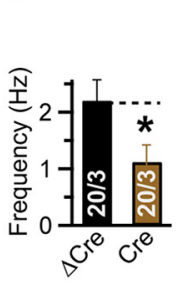

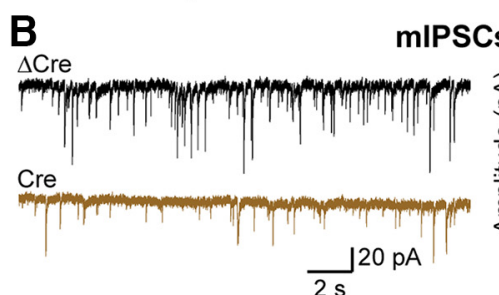

mIPSCs

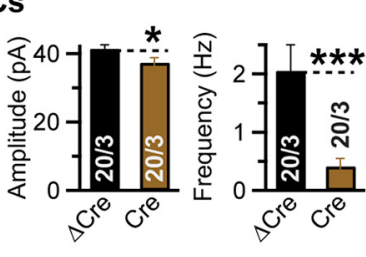

E
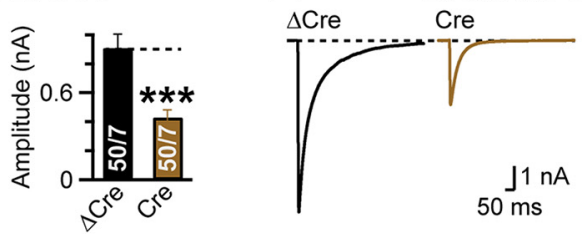
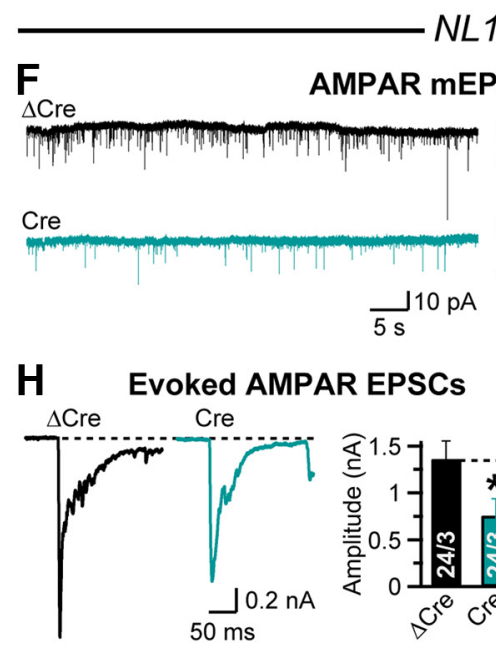

NL123 triph

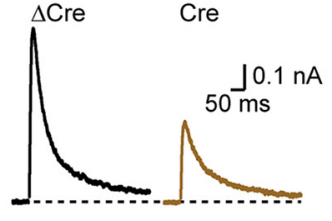

\section{Giple cKO - sparse cKO in transfected neurons
mIPSCs

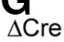

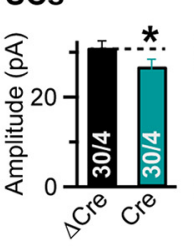

I

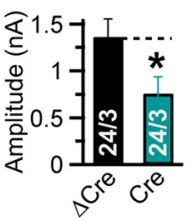

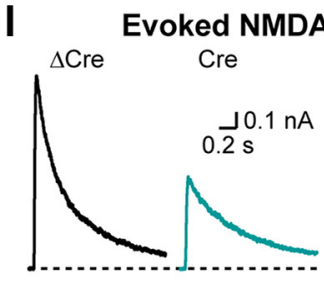
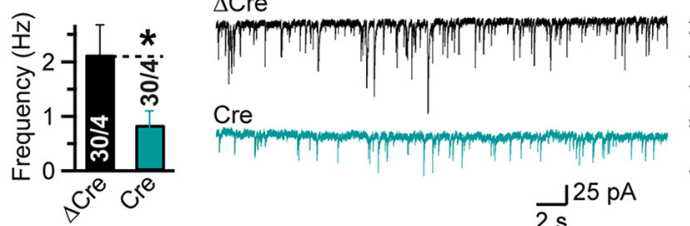

$\underset{2 s}{2} 25 \mathrm{pA}$

$\mathrm{J}$

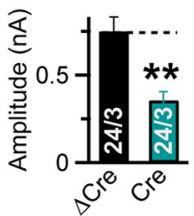

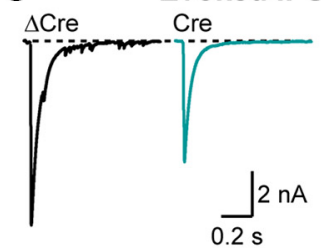

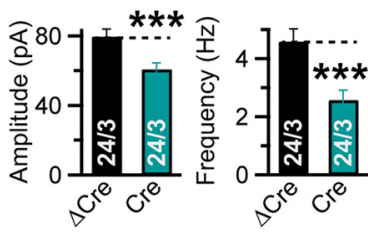

Evoked IPSCs

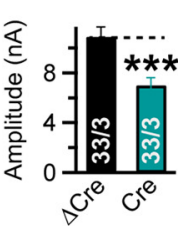

Figure 3. Acute triple KO of NL1, NL2, and NL3 (NL123) in cultured hippocampal neurons impairs excitatory and inhibitory synaptic strength by a cell-autonomous, postsynaptic mechanism. $\boldsymbol{A}, \boldsymbol{B}$, Acute lentiviral deletion of NL123 decreases the amplitudes and frequency of spontaneous $\mathrm{mEPSCS}(\boldsymbol{A})$ and $\mathrm{mIPSCS}(\boldsymbol{B})$. Left, representative traces; right, summary graphs of the $\mathrm{mEPSC} / \mathrm{mIPSC}$ amplitudes and frequency at DIV14-16. C-E, Acute lentiviral deletion of NL123 decreases the amplitudes of evoked synaptic responses in excitatory synapses (measured as AMPAR-mediated, $C$; and NMDAR-mediated EPSCS; $\boldsymbol{D}$ ) and in inhibitory synapses (IPS(S; $\boldsymbol{E}$ ). Left, Representative traces. Right, Summary graphs of the EPSC/IPSC amplitudes at DIV14-16. $\boldsymbol{F}-\boldsymbol{J}$, Same as $\boldsymbol{A}-\boldsymbol{E}$, except that hippocampal neurons cultured from NL123 triple cKO mice were sparsely transfected with plasmids encoding $\Delta$ Cre or Cre, as indicated. Data are reported as the mean \pm SEM; numbers of neurons/independent cultures analyzed are shown in bars. Significant differences between $\Delta$ Cre and Cre conditions are indicated with asterisks $\left({ }^{*} p<0.05\right.$; ${ }^{* *} p<0.01$; ${ }^{* * *} p<0.005 ;$ unpaired, one-tailed $t$ test).

LI-COR Odyssey CLx Imaging System. A mouse $\beta$-actin antibody (1:1000; Sigma-Aldrich; RRID: AB_476692) was used as a loading control for protein quantifications.

Electrophysiology. Patch-clamp experiments were performed essentially as described previously (Maximov et al., 2007; Chanda et al., 2013). In brief, primary neurons from hippocampal or cortical cells were patched with internal solution containing as follows (in mM): 135-145 $\mathrm{CsCl}_{2}, 0-5 \mathrm{NaCl}, 10$ HEPES, 1-5 EGTA, 1 Na-GTP, 1-5 QX-314, 4 MgATP, and $0.3 \mathrm{Na}_{2} \mathrm{GTP}$, with $\mathrm{pH}$ adjusted to 7.2-7.4 with $\mathrm{CsOH}$, and osmolarity of 305-310 mOsm. The external bath solution contained the following (in mM): $140-150 \mathrm{NaCl}, 4-5 \mathrm{KCl}, 2-3 \mathrm{CaCl}_{2}, 1 \mathrm{MgCl}_{2}, 10$ glucose, and 10 HEPES, with $\mathrm{pH}$ adjusted to 7.4 with $\mathrm{NaOH}$, and osmolarity of 305-315 mOsm. Individual receptor-mediated synaptic responses were isolated using pharmacological agents (picrotoxin; 50-100 $\mu \mathrm{M} ; \mathrm{GABA}_{\mathrm{A}} \mathrm{R} /$ GlycineR Blocker, Tocris Bioscience), CNQX (AMPAR blocker; 10-25 $\mu \mathrm{M}$; Tocris Bioscience), and DL-AP5 (NMDAR blocker; $50 \mu \mathrm{M}$; Tocris Bioscience) in the following specific combinations: picrotoxin plus AP5 for AMPAR-mediated evoked/mini EPSCs; picrotoxin plus CNQX for NMDAR-mediated evoked EPSCs; and AP5 plus CNQX for evoked/mini-IPSC measurements. Tetrodotoxin (TTX; $2 \mu \mathrm{M}$; Ascent Scientific) was additionally added to the external solution during all miniature EPSC (mEPSC) and mIPSC recordings to block presynaptic release induced by spontaneous action potentials. The AMPARmediated EPSCs and $\mathrm{GABA}_{\mathrm{A}} \mathrm{R}$-mediated IPSCs were recorded at a holding potential $\left(V_{\text {hold }}\right)$ of $-70 \mathrm{mV}$, whereas NMDAR-mediated EPSCs were measured at $+50 \mathrm{mV}$. The coefficient of variation $(\mathrm{CV})$ for AMPAR-mediated evoked EPSCs was calculated (SD/mean) from 8-20 consecutive trials. Paired-pulse ratios (PPRs) for NMDAR EPSCs and $\mathrm{GABA}_{\mathrm{A}} \mathrm{R}$ IPSCs were measured at a $100 \mathrm{~ms}$ time interval between two successive stimulation pulses. For use-dependent block of NMDAR EPSCs, stimulations of synaptic inputs were halted after achieving a stable baseline current at $V_{\text {hold }}=+50 \mathrm{mV}$, cells were then bath perfused with MK-801 (10 $\mu \mathrm{M}$; Tocris Bioscience) while maintaining a $V_{\text {hold }}=$ $-70 \mathrm{mV}$, and synaptic stimulations were resumed after 8-10 min for 100 successive pulses $(0.1 \mathrm{~Hz})$ at $V_{\text {hold }}=+50 \mathrm{mV}$; EPSC amplitudes from all trials were normalized to the first pulse after MK-801 application. Puff applications of AMPA (R-S AMPA hydrobromide, Tocris Bioscience; in the presence of $1 \mathrm{~mm} \gamma \mathrm{DGG}$, a low-affinity AMPAR competitive inhibitor; it additionally contained TTX plus picrotoxin plus AP5) and GABA (Tocris Bioscience; in the presence of $2 \mu \mathrm{M}$ CGP55845, a GABA $\mathrm{B}$ antagonist; it additionally contained TTX plus AP5 plus CNQX) were performed for $100 \mathrm{~ms}$ with 20 psi using a Picospritzer III (Parker Instrumentation), and the peak amplitude/total charge transfer was calculated within $30 \mathrm{~s}$ from puff application. 


\section{- Effect of global NL123 triple cKO on synapse density \& size -}

A
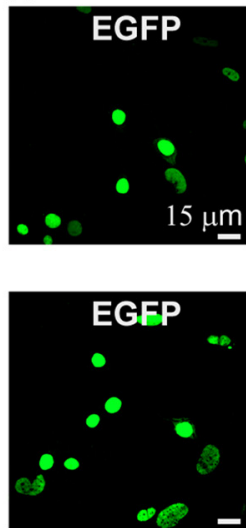

B
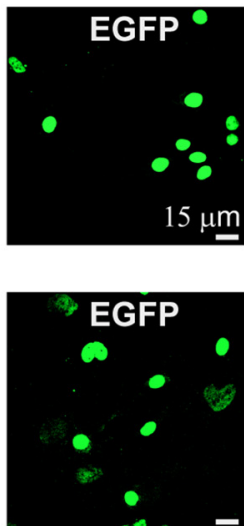

$\Delta$ Cre
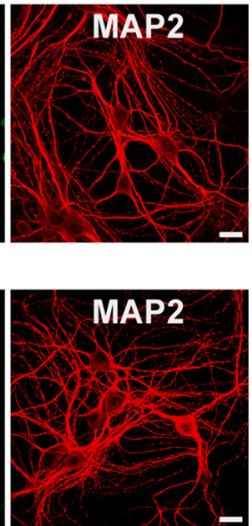

Cre
vGLUT1
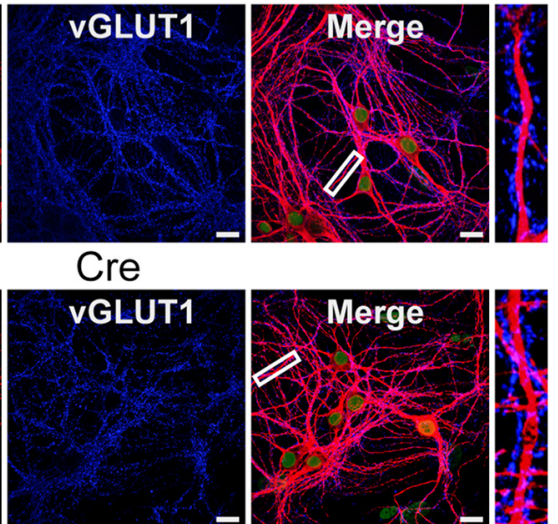

$\Delta$ Cre
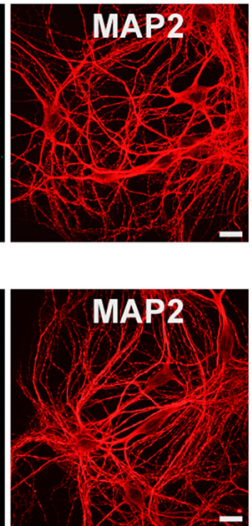

Cre
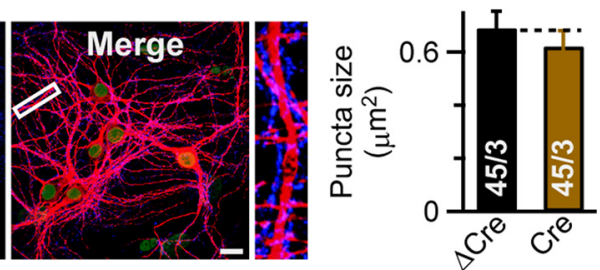

C Experimental procedure for spine density measurements
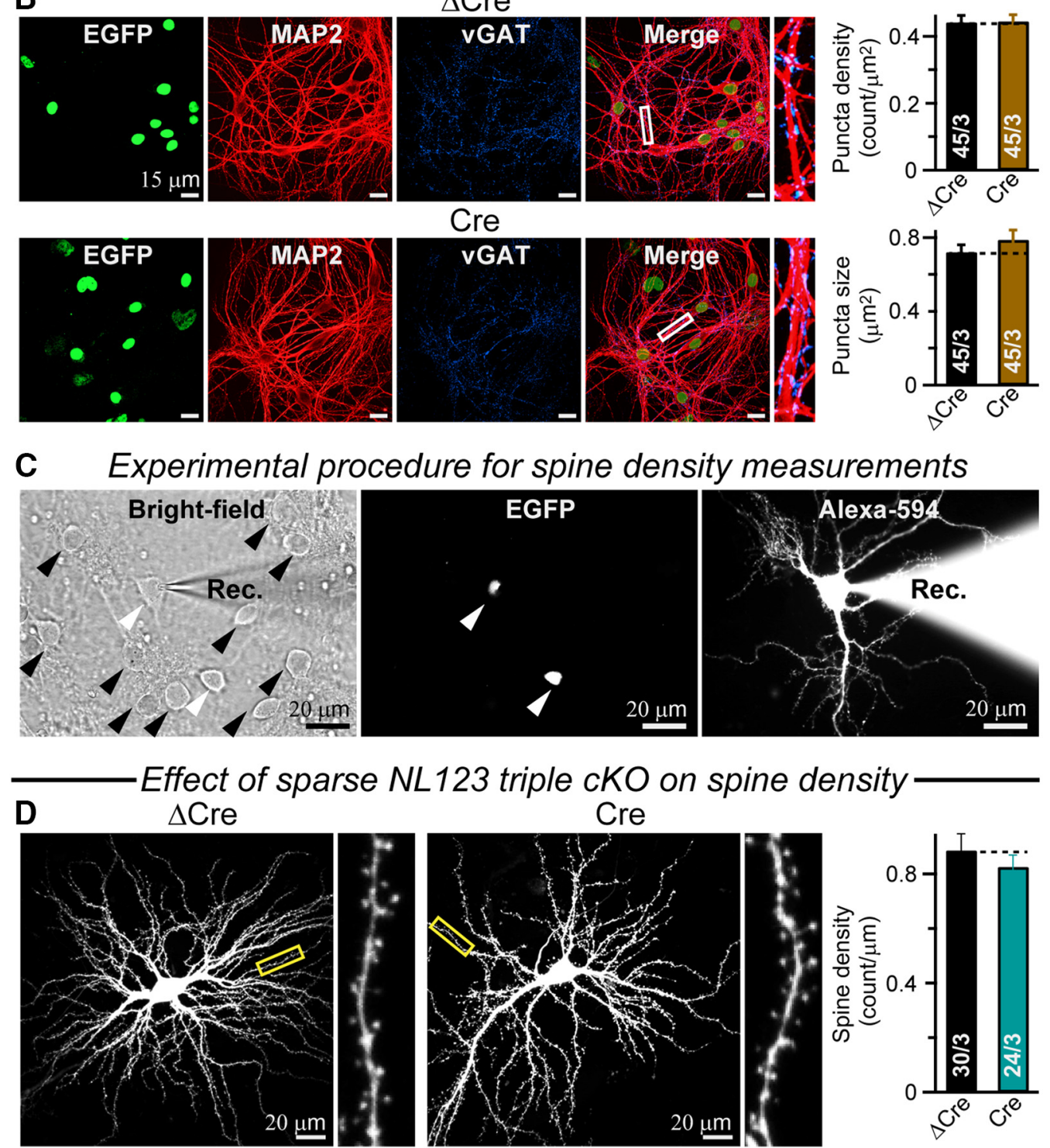

Figure 4. Acute $\mathrm{CKO}$ of neuroligins does not alter synapse or spine numbers in cultured hippocampal neurons. $\boldsymbol{A}$, Uniform deletion of neuroligins in all neurons does not decrease excitatory synapse density in hippocampal neurons cultured from NL123 triple cKO mice. Neurons were infected at DIV3 with lentiviruses expressing $\Delta$ Cre (top row) or Cre (bottom row), and immunostained at DIV14-16 for EGFP to identify nuclear $\Delta$ Cre/Cre expression, for MAP2 to label dendrites, and for vGLUT1 to mark excitatory synapses. Left, representative images as indicated (right view shows an expanded view of the selected dendrites boxed in the merged image); right, summary graphs of the density (top) or size (bottom) of vGLUT1-positive synaptic puncta. $\boldsymbol{B}$, Same as $\boldsymbol{A}$, except that neurons were immunostained for vGAT to mark inhibitory synapses. C, Neurons cultured from NL123 triple cKO mice were sparsely transfected at DIV3 with plasmids expressing $\Delta$ Cre-EGFP or Cre-EGFP, and patched at DIV14-16 with a recording pipette (Rec.) containing the fluorescent dye Alexa Fluor-594 to enable visualization of the entire dendritic (Figure legend continues.) 


\section{A Lentiviral NL123 triple cKO in cultured hippocampal neurons (DIV28) \\ $\Delta$ Cre}

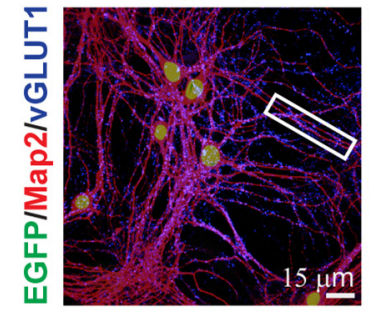

B

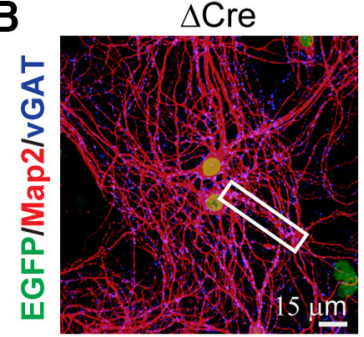

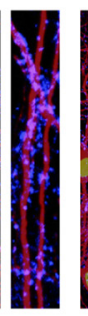

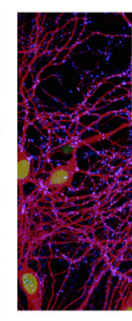
Cre
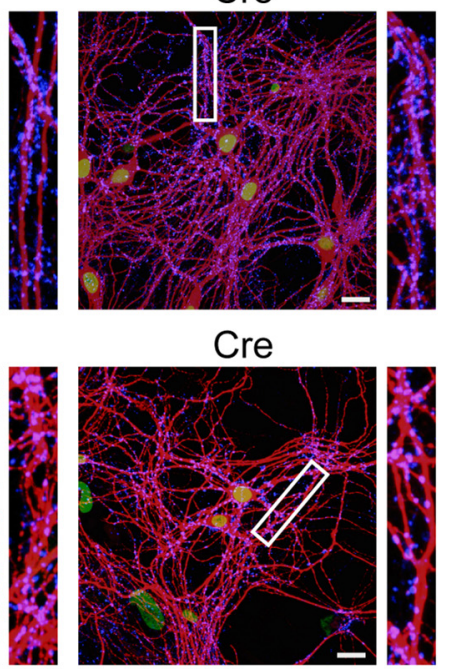
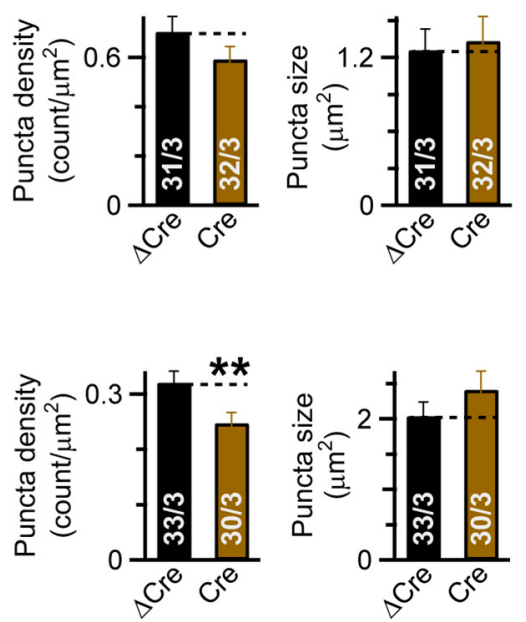

Figure 5. Extended deletion of NL123 for 4 weeks causes only minor change in synapse numbers. $A$, Representative images (areas identified with a white frame are enlarged on the right for each image) and summary graphs of the density (left) and size (right) of synaptic puncta of hippocampal neurons that were cultured from NL123 triple cK0 mice, infected at DIV3 with lentiviruses expressing $\Delta$ (re (control) or (re, and immunostained at DIV28 for vGLUT1 (to label excitatory synapses), MAP2 (to visualize dendrites), and EGFP (to assess infection efficiency since the lentiviruses express (re or $\Delta$ (re as EGFP fusion proteins). $\boldsymbol{B}$, Same as $\boldsymbol{A}$, except that neurons were stained for the inhibitory synapse marker vGAT instead of the excitatory synapse marker vGLUT1. Data are reported as the mean \pm SEM; total number of fields/batches analyzed is indicated in the bars. Statistical significance was assessed using an unpaired, one-tailed $t$ test $\left(^{* *} p>0.01\right.$; all other comparisons were $p>0.05)$.

Synaptic immunolabeling. For synapse number quantification, cells were washed with PBS and immediately fixed with 4\% PFA (Affymetrix) for $10 \mathrm{~min}$ at room temperature. Cells were next permeabilized and blocked in $0.1-0.2 \%$ Triton X-100 (Sigma-Aldrich) plus $10 \%$ cosmic calf serum (GE Healthcare Life Sciences) in PBS, followed by primary and secondary antibody incubation, for $1 \mathrm{~h}$ for each treatment, followed by four PBS washes. Coverslips were then mounted using Fluoromount-G (Southern Biotech), and images were acquired with an A1Rsi (Nikon) confocal microscope using $60 \times$ oil-objectives. Sequential acquisition was used to avoid bleed through between channels. The laser power, photomultiplier gain, and offset parameters were adjusted to avoid background signals and pixel saturation but were kept constant for all conditions within same experiment. Quantitative puncta-analysis for synapsin/vesicular GLUT1 (vGLUT1)/vesicular GABA transporter (vGAT) immunoreactivity was performed using ImageJ (National Institutes of Health; RRID: SCR_003070) software using maximum intensity projections of optical sections ( $7-13$ sections of $0.4-0.5 \mu \mathrm{m}$ thickness). All images within the same experiments were equally thresholded by intensity to exclude background signals, and only puncta number/size was estimated. For puncta density calculation, microtubule-associated protein 2 (MAP2) signals were used for area normalization. Antibodies used for these experiments were as follows: mouse anti-MAP2 (1:500; SigmaAldrich; RRID: AB_477171); E028 rabbit anti-synapsin (1:500; Südhof laboratory; RRID: AB_2315400); rabbit anti-vGLUT1 (1:1000; Synaptic

(Figure legend continued.) arbor and of spines of a transfected neuron (left, bright-field image, with white and black arrowheads indicating transfected and nontransfected neighboring neurons, respectively; center, EGFP fluorescence of transfected neurons expressing nuclear Cre- or $\Delta$ Cre-EGFP fusion proteins; right, Alexa Fluor-594 fluorescence of a patched transfected neuron). $\boldsymbol{D}$, Sparse deletion of neuroligins in a small subset of neurons does not decrease the spine density, as quantified with Alexa Fluor-594 fluorescence. Left and center, Representative images of Alexa Fluor-594-filled neurons (for better visualization, boxed dendritic sections are expanded on the right of each neuron), sparsely transfected with $\Delta$ (re vs (re-expressing vectors; right, summary graphs of the spine density. All summary data are the reported as the mean \pm SEM; numbers of fields/independent cultures analyzed are shown in bars. No significant differences ( $p>0.05$, unpaired, one-tailed $t$ test) were detected between $\Delta$ Cre vs Cre conditions in any of the experiments.
Systems; RRID: AB_2315552); rabbit anti-vGAT (1:1000; Synaptic Systems; RRID: AB_887869); chicken anti-GFP (1:1000; Aves Labs; RRID: AB_10000240); mouse anti-HA (1:1000; Covance; RRID: AB_291263); and Alexa Fluor-488-, Alexa Fluor-555-, and Alexa Fluor-647conjugated secondary antibodies (Life Technologies). For spinecounting experiments, cells were perfused for 5-10 min with Alexa Fluor-594 (0.5 mM; Life Technologies) using a patch pipette and then fixed with PFA and mounted with Fluoromount-G. Spine density analysis was performed using Neuron Studio software (Rodriguez et al., 2008) with manual curation. For depolarization-induced presynaptic uptake of anti-Synaptotagmin-1 luminal antibody (Syt $1_{\text {lum }}$; rabbit; Synaptic Systems; RRID: AB_887835), cells were briefly incubated for 10 min with fresh Neuobasal plus B27 media (containing $1.8 \mathrm{~mm} \mathrm{Ca}^{2+}$; Thermo Fisher Scientific) plus diluted Syt $1_{\text {lum }}$ antibody $(1: 100)$ and $50 \mathrm{~mm} \mathrm{KCl} \mathrm{in}$ the presence of TTX $(2 \mu \mathrm{M})$ plus CNQX $(25 \mu \mathrm{M})$ plus AP5 $(50 \mu \mathrm{M})$ plus picrotoxin $(50 \mu \mathrm{M})$. Cells were then immediately washed with PBS and fixed with PFA, permeabilized with Triton X-100, and coimmunostained with mouse anti-synapsin-1 (Syn1) antibody (1:1000; Synaptic Systems; RRID: AB_887805). Experiments for surface GluA2 immunostaining and GluA2-vGLUT1 colocalization assay were performed essentially as described by Chanda et al. (2016). In brief, fixed but nonpermeabilized cells were first immunolabeled with mouse GluA2 antibody (1:50; Millipore; RRID: AB_2113875) then washed with PBS, permeabilized with Triton X-100, and stained with vGLUT1 antibody (1:1000). Colocalization analysis was performed using the JACoP plugin in ImageJ software.

FM1-43 assay for presynaptic exocytosis. Low-density primary hippocampal cultures from NL123 triple cKO animals were globally infected at DIV3 with lentivirus expressing $\Delta$ Cre versus Cre-EGFP. At DIV14-16, cells were labeled with FM1-43 dye ( $5 \mu \mathrm{M}$; Life Technologies) for $90 \mathrm{~s}$ with an external recording solution containing $50 \mathrm{~mm} \mathrm{KCl}$. Activitydependent recycling terminals loaded with FM1-43 were visualized after $10-15$ min of perfusion with a $\mathrm{KCl}$-free and dye-free regular external solution. Neurons were then exposed to a dye-free external solution containing $50 \mathrm{~mm} \mathrm{KCl}$ for depolarization-induced vesicle depletion. All solutions contained $3 \mathrm{mM} \mathrm{CaCl}_{2}$ and the inhibitor cocktail TTX $(2 \mu \mathrm{M})$ plus CNQX $(25 \mu \mathrm{M})$ plus AP5 $(50 \mu \mathrm{M})$ plus picrotoxin $(50 \mu \mathrm{M})$ to avoid $\mathrm{Na}^{+}$channel and/or recurrent synaptic activity-mediated presynaptic depolarization. Images were acquired every second using a Rolera-XR digital CCD camera (Qimaging), and an Olympus BX51WI microscope 
NL123 triple cKO - global cKO in lentivirally infected neurons

A Activity-dependent presynaptic endocytosis
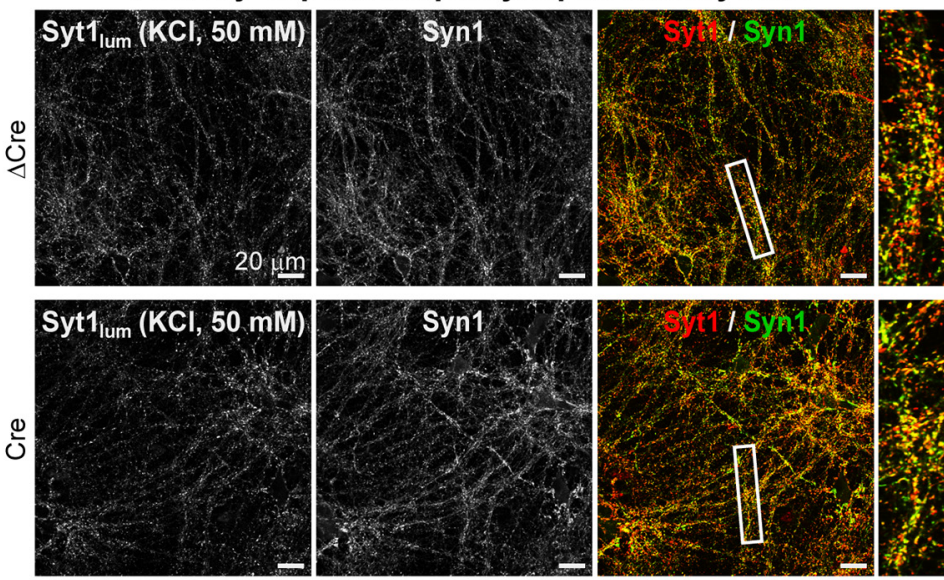

C

Activity-dependent presynaptic exocytosis
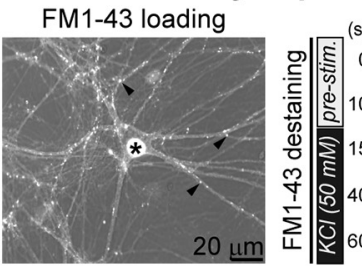

$\Delta \mathrm{Cr}$
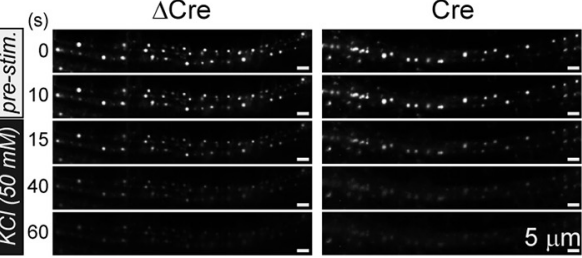

\section{D}

FM1-43 destaining kinetics

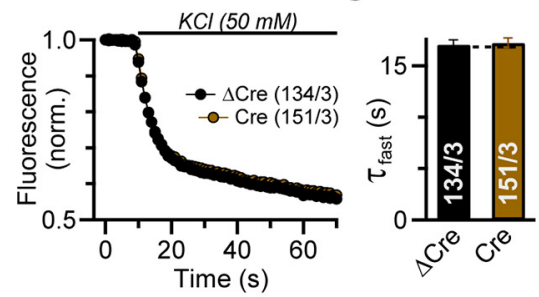

E AMPAR EPSC variability
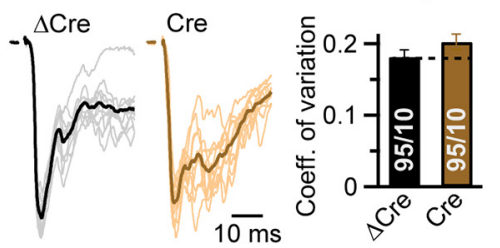

F NMDAR EPSC paired-pulse

G IPSC paired-pulse

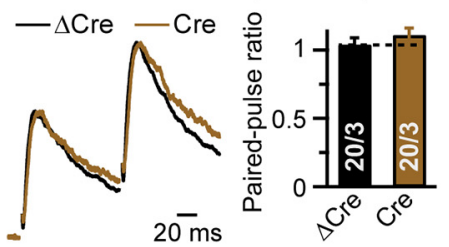

Use-dependent inhibition of NMDAR

H
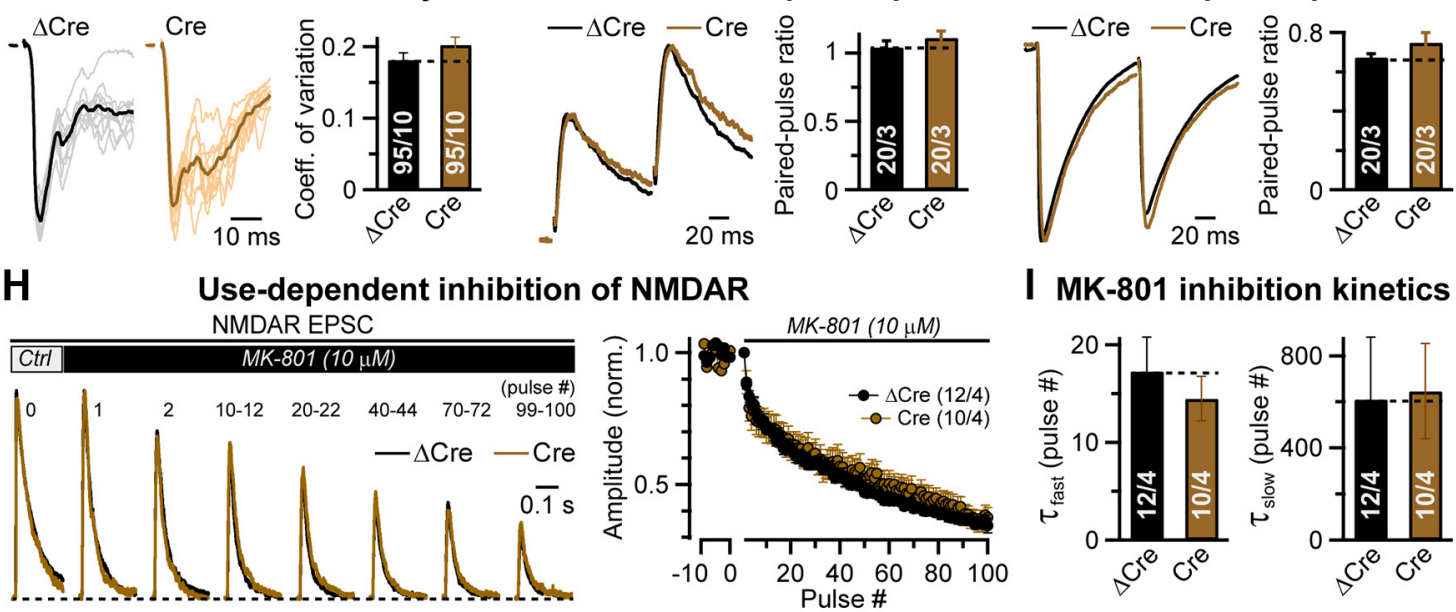

I MK-801 inhibition kinetics

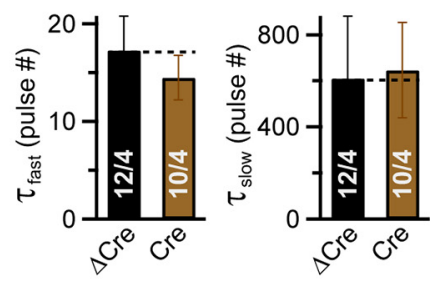

NL123 triple cKO - sparse cKO in transfected neurons

J AMPAR EPSC variability

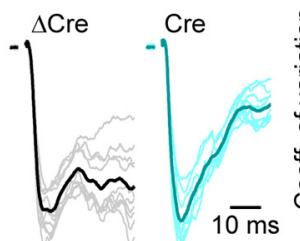

$\mathrm{K}$

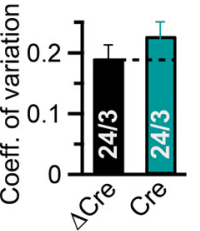

$K$ NMDAR EPSC paired-pulse

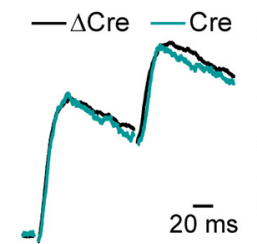

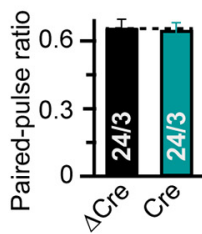

L IPSC paired-pulse

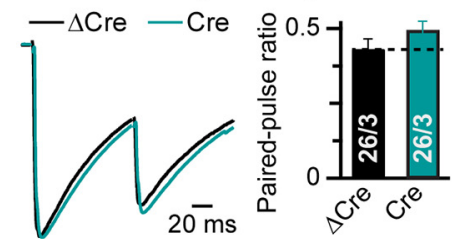

Figure 6. Conditional loss of neuroligins does not affect the properties of presynaptic neurotransmitter release. $A$, Representative images (Pseudo colored) of dendritic sections from hippocampal

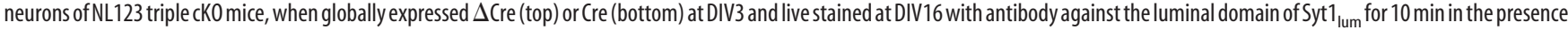
of $50 \mathrm{~mm} \mathrm{KCl}$ and $1.8 \mathrm{~mm} \mathrm{Ca}^{2+}$ (left), and coimmunostained for Syn1 (middle) after fixation and permeabilization (signals merged, right). Insets are magnified views of the boxed areas (white) from merged images. $\boldsymbol{B}$, Average graphs indicate the size (top left) and density (top right) of Syt1-positive actively recycling presynaptic boutons, and the colocalization of Syt1-labeled active synapses with Syn1-positive presynaptic terminals (bottom left) or the colocalization of Syn1-positive presynaptic puncta with Syt1-positive terminals (bottom right). Colocalizations are measures of normalized Mander's coefficients for respective correlations of immunofluorescence signals from $\Delta$ (re (black) vs (re (brown) conditions. $\boldsymbol{C}$, Experimental strategy (left) to directly monitor presynaptic vesicle release in primary hippocampal neurons derived from NL123 triple cK0 mice, which were globally infected at DIV3 with lentivirus-expressing EGFP-tagged $\Delta$ Cre or Cre (asterisk), loaded with $5 \mu \mathrm{m}$ FM1-43 dye for $90 \mathrm{~s}$ in the presence of $50 \mathrm{~mm} \mathrm{KCl}$ and $3 \mathrm{~mm} \mathrm{Ca}^{2+}$ at DIV14-16, and fluorescently labeled presynaptic boutons (arrowheads) (Figure legend continues.) 
equipped with filter sets (41017 bandpass filter, Chroma; Excitation wavelength, $470 \pm 20 \mathrm{~nm}$; Emission wavelength, $525 \pm 25 \mathrm{~nm}$ ) that allowed fluorochrome detection of both nuclear EGFP and synaptic FM1-43 signals from the same cell. Images were analyzed using ImageJ software, background subtracted, and corrected for photo-bleaching using nonsynaptic areas, and average intensities were normalized to the initial image $10 \mathrm{~s}$ before $\mathrm{KCl}$ stimulation.

Data presentation. All average data in bar graphs represent the mean \pm SEM. For most experiments, statistical comparisons between Cre and $\Delta$ Cre conditions were made using an unpaired, one-tailed, Student's $t$ test, except for the quantitative PCR and Western blot analysis (Fig. $2 C, E)$, where a paired $t$ test was conducted. For multiple comparisons (Fig. 10), one-way ANOVA with post hoc Tukey's test was performed.

\section{Results}

\section{Time course of synapse formation in cultured neurons}

In order to assess the synaptic role of neuroligins, it is important to first understand the developmental time course of synaptogenesis in the cultured neurons used, because this time course may vary considerably between culture preparations. Therefore, we cultured hippocampal neurons from newborn mice and monitored their morphological and functional synaptic properties every other day from DIV4 to DIV16 (Fig. 1A). Over this time period, we observed a gradual increase in the complexity of neurite outgrowth, a continuous increase in membrane capacitance $\left(\mathrm{C}_{\mathrm{m}}\right)$, and a steady decrease in input resistance $\left(\mathrm{R}_{\mathrm{m}}\right.$; Fig. $\left.1 A, B\right)$. Similarly, synapse density and synapse size also increased substantially from DIV4 to DIV16 (Fig. $1 C, D$ ). We then evaluated the functional maturation of excitatory versus inhibitory synapses and measured AMPAR-mediated mEPSCs and $\mathrm{GABA}_{\mathrm{A}} \mathrm{R}$-mediated mIPSCs (Fig. 1E,F). Both mEPSCs and mIPSCs increased in amplitude and frequency over time, as expected. However, mEPSCs matured much faster than mIPSCs and reached a maximal steady-state level by DIV10 to DIV12 (Fig. 1E). The mIPSCs, in contrast, increased at a much slower rate and did not saturate even at DIV16 (Fig. 1F). Together, these data suggest that synaptogenesis proceeds continuously within $4-16 \mathrm{~d}$ in cultured hippocampal neurons, with excitatory synapses developing more

\section{$\leftarrow$}

(Figure legend continued.) on dendritic branches were visualized after a 10 min wash with dye-free media. The time-lapse images (right) represent FM1- 43 signals from presynaptic terminals of NL123 triple cKO hippocampal neurons expressing $\Delta$ Cre or Cre as indicated, before (pre stim., top two) and after exposure to $50 \mathrm{~mm} \mathrm{KCl}$ in the presence of $3 \mathrm{~mm} \mathrm{Ca}^{2+}$ (bottom three). $\boldsymbol{D}$, Normalized average intensity of FM1-43 signals (left) from $\Delta$ (re (connected filled circles, black) vs (re (connected filled circles, brown) expressing NL123 cKO neurons, before and after $50 \mathrm{~mm} \mathrm{KCl} \mathrm{treatment} \mathrm{(black} \mathrm{bar).} \mathrm{Data} \mathrm{from} \mathrm{individual} \mathrm{cells} \mathrm{were} \mathrm{further} \mathrm{fitted} \mathrm{to} \mathrm{a} \mathrm{double-}$ exponential function, and the summary graph (right) indicates the fast-decay component of FM1-43 signal intensity following $50 \mathrm{~mm} \mathrm{KCl} \mathrm{exposure} \mathrm{for} \Delta$ Cre (black) and Cre (brown) conditions. $E$, Analysis of variability in AMPAR-mediated evoked EPSCs for NL123 CKO hippocampal neurons globally expressing $\Delta$ Cre vs Cre. Example traces (left) show 10 consecutive trials (light shades) overlaid with average values (dark shades). Summary graph (right) depict coefficients of variation as an indirect measure of release probability. $F, G$, Representative traces (left) and summary graphs (right) of average PPRs ( $\Delta t=100 \mathrm{~ms}$ ) of NMDAR-mediated EPSCs (F) or IPSCs (G). $\boldsymbol{H}$, Sample traces (left) and average amplitudes (right) of NMDAR-mediated evoked EPSCS during repetitive synaptic stimulation at $0.1 \mathrm{~Hz}$ from NL123 CKO neurons globally expressing $\Delta$ Cre (black) or Cre (brown), before (Ctrl) and after bath application of $10 \mu \mathrm{M}$ MK-801 (black bar), as normalized to trial 1 in MK-801. I, Summary graphs indicate fast (left) and slow (right) kinetic components for MK-801-dependent inhibition of NMDAR-mediated evoked EPSC amplitudes, when fitted with a double-exponential function. $\boldsymbol{J}-\boldsymbol{L}$, Same as $\boldsymbol{E}-\boldsymbol{G}$, except for NL123 triple cKO hippocampal neurons sparsely transfected with plasmids encoding $\Delta C$ re or $\mathrm{Cre}$, as indicated. All average data are reported as the mean $\pm \mathrm{SEM}$; corresponding numbers of fields imaged (for $\boldsymbol{A}$ and $\boldsymbol{B}$ ), synaptic terminals analyzed (for $\boldsymbol{C}$ and $\boldsymbol{D}$ ), or cells patched $(\boldsymbol{E}-\boldsymbol{L})$ per independent experimental batches are indicated. No significant difference $(p>0.05$, unpaired, one-tailed $t$ test) between $\Delta$ (re and (re conditions was detected for any of the experiments. rapidly than inhibitory synapses, as observed in vivo for the visual cortex (Sutor and Luhmann, 1995).

\section{Cre-recombinase expression efficiently deletes neuroligins in cKO neurons}

To evaluate the cellular effects of deletions of endogenous neuroligins, we next cultured hippocampal neurons from various neuroligin cKO mice (NL1 single cKO, NL2 single cKO, NL3 single cKO, NL13 double cKO, NL23 double cKO, and NL123 triple $\mathrm{cKO}$ ). We infected the neurons at DIV3 with lentiviruses expressing EGFP-fused nuclear Cre-recombinase (which generates a global loss of selective neuroligin isoforms in all neurons due to high infection efficiency; Fig. $2 A, B$ ) before synaptogenesis and synaptic activities were observed (see Fig. 1). A nonfunctional mutant version of the Cre-recombinase $(\Delta \mathrm{Cre})$ was used in all experiments as a control. Global expression of Crerecombinase caused a nearly complete loss of the respective neuroligin mRNAs and protein expression in corresponding NL cKO neurons, as analyzed at DIV14-16 (Fig. 2C-E). Crerecombinase was also effective in NL123 triple cKO neurons, demonstrating that even having six floxed alleles does not impede recombination. Therefore, all of our following experiments for neuroligin cKO analyses were performed at DIV14-16 after Cre-recombinase expression at DIV3, except when otherwise stated (Figs. 5, 14). For some experiments (Figs. $3 F-J, 4 C, D, 11 D, 12 F-J, 13 C, D)$, we also delivered the lentiviral vectors using a calcium phosphate transfection protocol, which resulted in a sparse expression pattern of $\Delta$ Cre or Cre only in a few, well isolated neurons that could be visually separated from nontransfected neighboring cells (Fig. $2 F$ ).

\section{Endogenous neuroligins support general synapse function}

To evaluate the overall contribution to synaptic transmission of the three major neuroligin isoforms (NL1, NL2, and NL3; note that NL4 is not significantly expressed in cultured hippocampal neurons), we first analyzed triple NL123 cKO neurons. Lentivirus-mediated global loss of neuroligins caused a large reduction in both excitatory and inhibitory synaptic transmission, including the mEPSC amplitude and frequency (Fig. $3 A, \sim 25 \%$ and $\sim 50 \%$ decrease, respectively), mIPSC amplitude and frequency (Fig. $3 B, \sim 10 \%$ and $\sim 80 \%$ decrease, respectively), AMPAR-evoked EPSCs (Fig. $3 C, \sim 30 \%$ decrease), NMDAR-evoked EPSCs (Fig. 3D, $\sim 60 \%$ decrease), and $\mathrm{GABA}_{\mathrm{A}} \mathrm{R}$-evoked IPSCs (Fig. $3 E, \sim 70 \%$ decrease). These data suggest that endogenous neuroligins play a key role in determining both excitatory and inhibitory synaptic strengths.

We next asked whether the neuroligin deficiency phenotype, although already severe, might be even more pronounced upon sparse deletions of neuroligins, after which a few neuroligindeficient neurons would have to compete with surrounding wildtype neurons for synaptic contacts. We sparsely transfected NL123 triple cKO hippocampal neurons with expression vectors for $\Delta$ Cre-EGFP as a control or for Cre-EGFP (Fig. $2 F$ ). Synaptic recordings from transfected cells demonstrated that the loss of neuroligins again significantly impaired mEPSC amplitude and frequency (Fig. $3 F, \sim 10 \%$ and $\sim 60 \%$ decrease, respectively), mIPSC amplitude and frequency (Fig. $3 G, \sim 20 \%$ and $\sim 50 \%$ decrease, respectively), AMPAR-evoked EPSCs (Fig. $3 H, \sim 50 \%$ decrease), NMDAR-evoked EPSCs (Fig. 3I, $\sim 60 \%$ decrease), and $\mathrm{GABA}_{\mathrm{A}} \mathrm{R}$-evoked IPSCs (Fig. 3J, $\sim 30 \%$ decrease). Because Crerecombinase expression by sparse transfection of NL123 triple cKO neurons produced a phenotype similar to that produced by the global infection of all neurons by lentiviral infection (Fig. 
A AMPAR synaptic distribution (NL123 triple cKO)
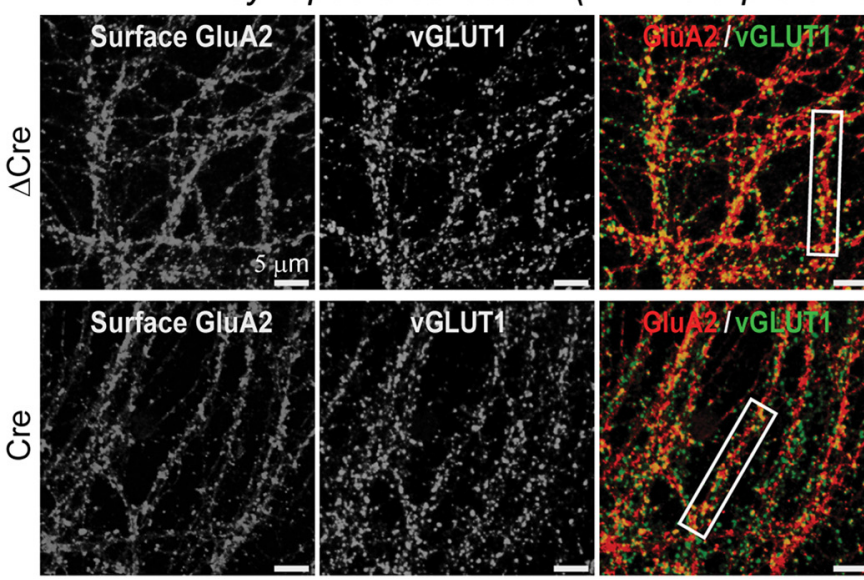

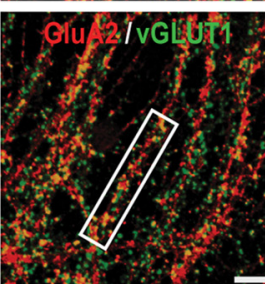

B AMPAR synaptic puncta

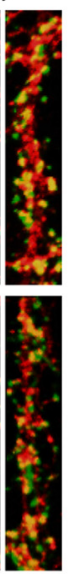
size \& density

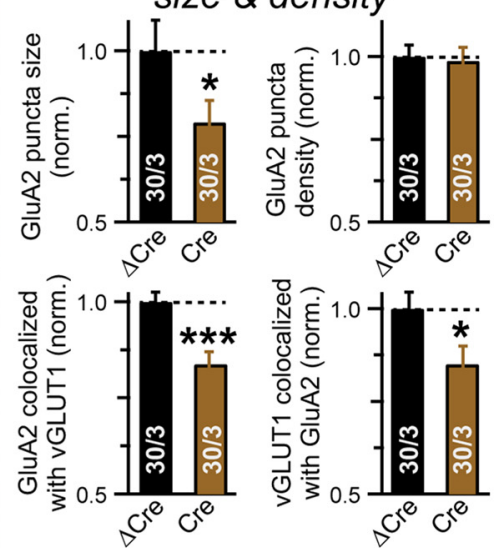

Measurements of agonist-application induced postsynaptic currents
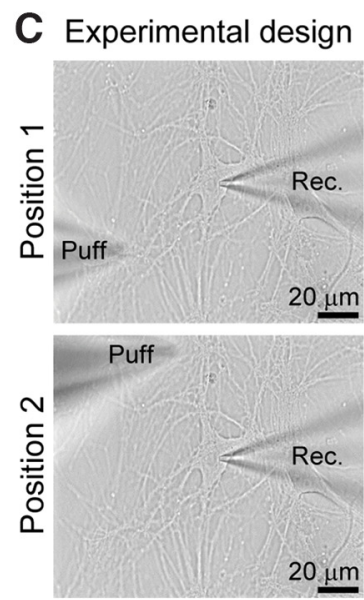

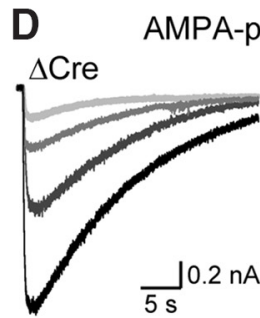

Cre

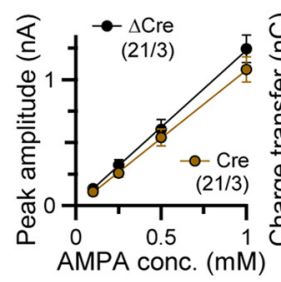

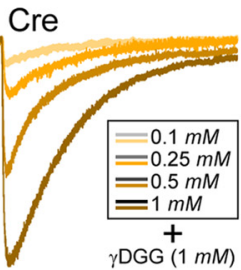

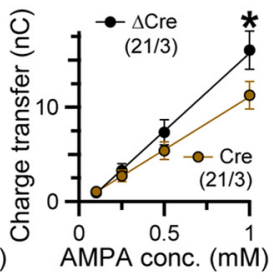

E
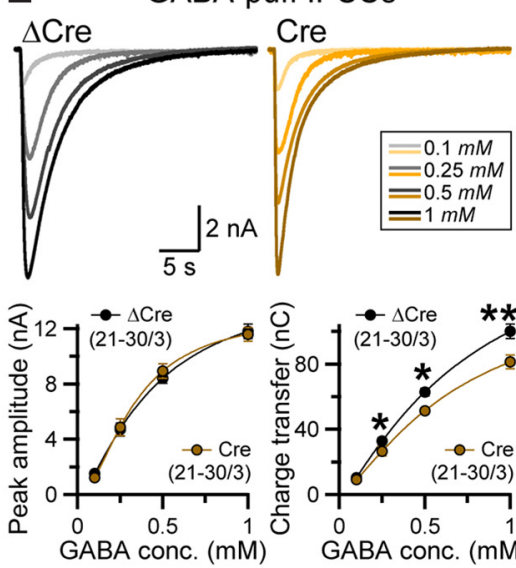

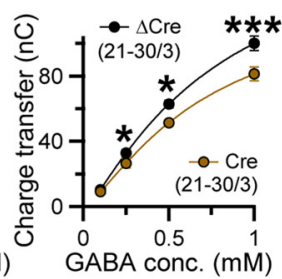

Figure 7. Global triple NL123 cKO decreases the synaptic and surface localizations of postsynaptic receptors. $\boldsymbol{A}$, Representative images (Pseudo colored) from dendritic branches of hippocampal neurons that were cultured from NL123 triple cK0 mice and infected with lentiviruses expressing $\Delta$ (re (top) or Cre (bottom) at DIV3 and immunostained for surface AMPARs (GluA2 staining, left) and excitatory synapses (vGLUT1 staining, center) at DIV16 (merged views, right). B, Summary graphs represent the size (top left) and density (top right) of GluA2-positive synaptic puncta, as well as the colocalization of GluA2 receptors with vGlut1-positive presynaptic terminals (bottom left) or the colocalization of vGlut1-positive presynaptic terminals with GluA2 receptor-positive puncta (bottom right). In these analyses, colocalizations are measured as normalized Mander's coefficients for respective correlations of immunofluorescence signals. $\boldsymbol{C}$, Sample image of a hippocampal neuron from low-density culture, as cells were patched using a recording pipette (Rec.), and a second pipette (Puff) was used to pressure deliver the selective receptor agonist near two different dendritic processes (Position 1 and Position 2). D, Example traces (top) of postsynaptic currents from NL123 triple cK0 neurons expressing $\Delta$ Cre (black) vs (re (brown), as induced by exogenous application of AMPA of varying concentrations (color shades) to selectively stimulate surface AMPARs in the presence of $\gamma D G G$ (1 mM, low-affinity competitive AMPAR antagonist), and summary graphs of the average peak amplitude (bottom left) and total charge transfer (bottom right) for dose-dependent AMPA-induced responses (AMPA conc.). For every cell, agonist-induced EPSC 5 were averaged for two to three different puff-pipette positions (see $\boldsymbol{C}$ ). Summary data are fit with straight lines; for peak amplitude, slope $=1.22 \pm 0.11$ (for $\Delta$ (re, black) and $1.08 \pm 0.1$ (for $(\mathrm{re}, \mathrm{brown}$ ); for total charge transfer, slope $=16.48 \pm 1.86$ (for $\Delta$ (re, black) and $11.21 \pm 1.41$ (for Cre, brown). $\boldsymbol{E}$, Same as $\boldsymbol{D}$, but for responses induced by puffs of GABA. Lines in summary graphs are fits with Hill equation (see legend to Fig. $1 B$ ); for peak amplitude, $P_{\max }=15.54 \pm 5.04 \mathrm{nA}$ (for $\Delta$ (re, black) and $13.1 \pm 2.13 \mathrm{nA}$ (for Cre, brown), $n=1.41 \pm 0.83$ (for $\Delta$ Cre, black) and $1.85 \pm 0.92$ (for Cre, brown), and $\mathrm{K}_{1 / 2}=435.65 \pm 144 \mu \mathrm{m}$ (for $\Delta$ Cre, black) and $329.8 \pm 51.6 \mu \mathrm{m}$ (for Cre, brown); for total charge transfer, $P_{\max }=180 \pm 108 \mathrm{nC}$ (for $\Delta$ Cre, black) and $130.43 \pm 67.4 \mathrm{nC}$ (for Cre, brown), $n=1.19 \pm 0.6$ (for $\Delta$ (re, black) and $1.35 \pm 0.73$ (for Cre, brown), and K ${ }_{1 / 2}=804.47 \pm 721 \mu \mathrm{m}$ (for $\Delta$ (re, black) and $690.22 \pm 479 \mu \mathrm{m}$ (for Cre, brown). Data in bar graphs are reported as the mean \pm SEM; numbers on the bars indicate total number of fields analyzed (for imaging) or cells recorded (for electrophysiology)/number of independent cultures, from each condition. Asterisks indicate significant differences between $\Delta$ Cre and Cre conditions ( ${ }^{*} p<0.05$; ${ }^{* * *} p<0.005$; unpaired, one-tailed $t$ test).

$3 A-E)$, our data suggest that the functions of neuroligins operate by a cell-autonomous mechanism.

Conditional loss of endogenous neuroligins does not affect synapse formation

The effect of the NL123 triple conditional deletion on synaptic function could be due either to an overall loss of synapse numbers or to presynaptic and/or postsynaptic functional impairments. To differentiate among these three possibilities, we first tested the effect of the NL123 triple deletion on synapse and spine numbers (Fig. 4).

We infected cultured hippocampal NL123 triple cKO neurons with $\Delta$ Cre-EGFP- or Cre-EGFP-expressing lentiviruses and stained the neurons with antibodies to MAP2 as a dendritic marker and to vGLUT1 or vGAT as markers of excitatory and inhibitory synapses, respectively. We then quantified the density and size of synaptic puncta but failed to uncover any significant difference between control ( $\Delta$ Cre $)$ and NL123-deficient neurons (Cre; Fig. $4 A, B$ ). Furthermore, to study the effect of the NL123 triple deletion on spines, we sparsely transfected cultured hippocampal NL123 cKO neurons with $\Delta$ Cre-EGFP- or Cre-EGFP-expressing plasmids, filled transfected neurons with the fluorescent dye Alexa Fluor-594 via a patch pipette at DIV14-16, and analyzed the spine density of the imaged neurons (Fig. 4C). Again, we observed no significant effect of the NL123 triple deletion on spine 


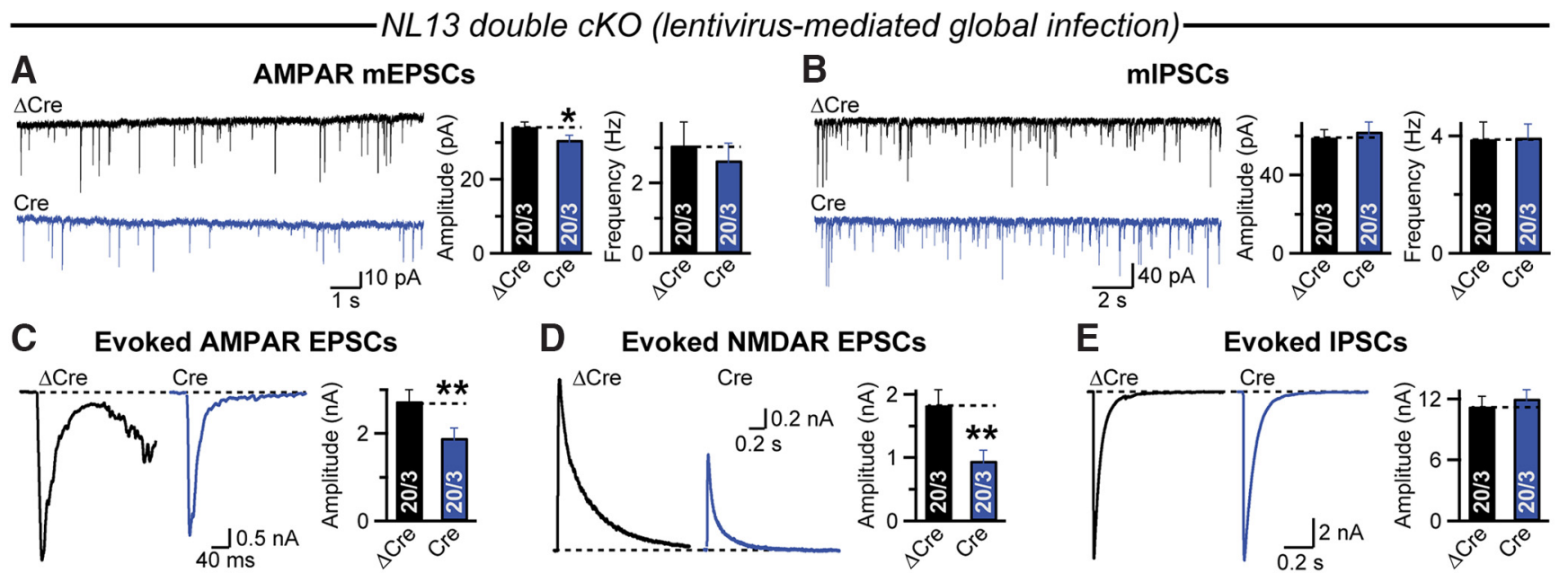

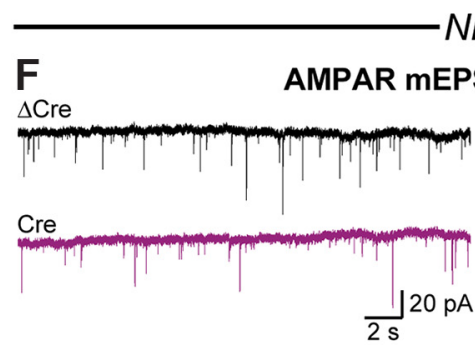

H Evoked AMPAR EPSCs
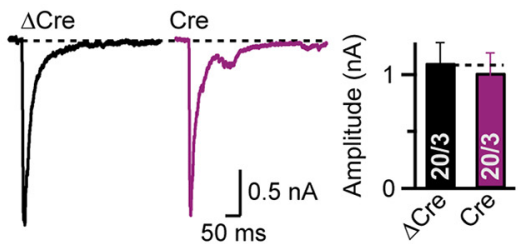

$N L 23$

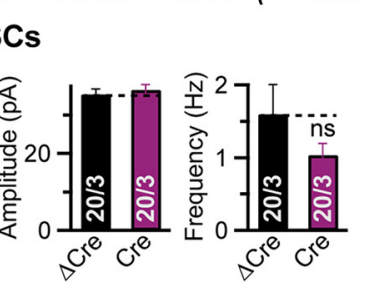

I

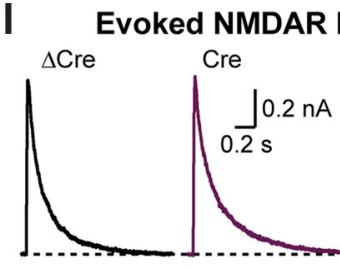

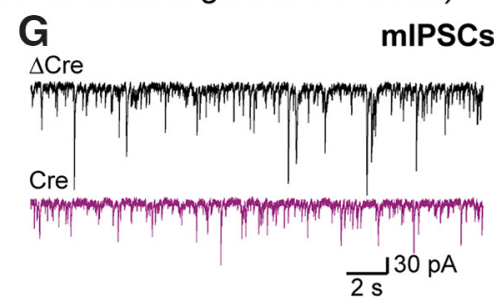

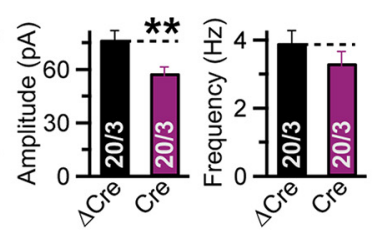

J

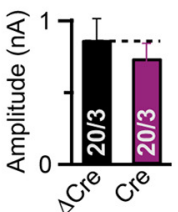

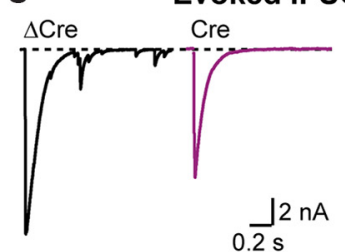

Figure 8. Acute conditional double cKO of neuroligins NL13 and of NL23 partially replicate the phenotype produced by conditional NL123 triple cK0 in cultured hippocampal neurons. A, Sample traces of AMPAR-mediated mEPSCS (left) recorded from DIV 14 to DIV16 primary hippocampal neurons of NL13 double cK0 animals, globally infected with lentivirus expressing $\Delta$ Cre (top) or Cre (bottom) at DIV3. Bar graphs (mean \pm SEM) represent the average amplitude (left) and frequency (right) of mEPSC events for $\Delta$ Cre (black) vs (re (blue) conditions. $\boldsymbol{B}$, Same as $\boldsymbol{A}$, except for GABAergic mIPSCS. $\boldsymbol{C}-\boldsymbol{E}$, Representative traces of evoked synaptic currents for AMPAR EPSCS (C) NMDAR EPSCS (D), and GABA $A_{A} R$ IPSCS $(\boldsymbol{E})$ recorded from $\Delta$ Cre-expressing (black) vs $($ re-expressing (blue) NL13 double cKO neurons. Corresponding bar graphs (mean \pm SEM) indicate average amplitudes of evoked EPSCS/PSCS for $\Delta$ Cre vs Cre condition. $\boldsymbol{F}-\boldsymbol{J}$, Same as $\boldsymbol{A}-\boldsymbol{E}$, except cultures were derived from NL23 double cK0, and infected with $\Delta$ Cre-expressing (black) or Cre-expressing (purple) lentivirus. Numbers on the bar graphs represent the total number of cells recorded/number of batches, for all experimental conditions. Significant differences between $\Delta$ Cre and Cre conditions are indicated with asterisks $\left({ }^{*} p<0.05\right.$; ${ }^{* *} p<0.01$; unpaired, one-tailed $t$ test). ns, Not significant. $(p>0.05)$.

numbers (Fig. 4D). Thus, the decrease in synaptic transmission induced by deletion of neuroligins is not due to a decrease in synapse or spine numbers.

It is possible that the decrease in synapse numbers observed upon the deletion of neuroligins in some experiments (Chih et al., 2005; Shipman et al., 2011; Kwon et al., 2012) may have been a secondary consequence of the chronic impairment in synaptic transmission. To test this hypothesis, we examined the effect of the NL123 triple deletion on synapse density after a longer time in culture (i.e., at DIV28 instead of DIV14-16; Fig. 5). Again, we detected no significant difference in excitatory synapse density between control and triple NL123 KO neurons (Fig. 5A). We did, however, observe a small but significant reduction in inhibitory synapse density in NL123-deficient neurons after 4 weeks of culture (Fig. 5B). These data suggest that prolonged loss of neuroligins may cause minor reductions in inhibitory synapse numbers, at least in a subset of synapses depending on the cultured cell types.
Conditional loss of NL123 does not alter the functional parameters of presynaptic release

The decreases in MEPSC and mIPSC amplitudes in NL123 triple cKO neurons indicate a reduction in postsynaptic AMPARs and GABARs, whereas the decreases in MEPSC and MIPSC frequencies could result from either presynaptic decreases in release probability or silencing of functional synapses, or postsynaptic decreases in neurotransmitter reception (see Fig. 3). To investigate whether the synaptic phenotypes in neuroligin-deficient neurons could be explained by a presynaptic mechanism, we tested activity-dependent vesicle reuptake in NL123 triple cKO neurons globally expressing $\Delta$ Cre versus Cre, using depolarization-induced endocytosis of an Syt1 luminal domain antibody (Matteoli et al., 1992). We measured the puncta size and density of Syt1-positive actively recycling terminals and also analyzed their colocalizations with Syn1-expressing presynaptic boutons but found no significant change in neuroligin-deficient cells for any of the parameters tested (Fig. 6A,B). Moreover, the 
NL1 single cKO (lentivirus-mediated global Cre-infection)

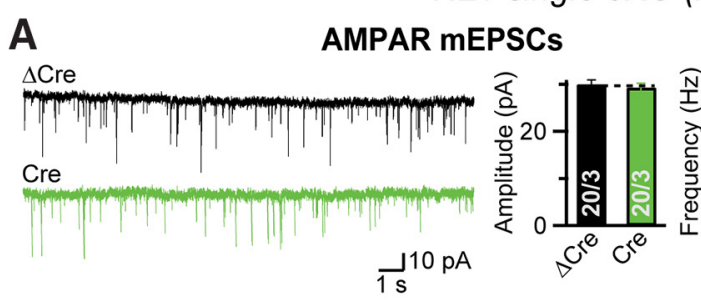

C
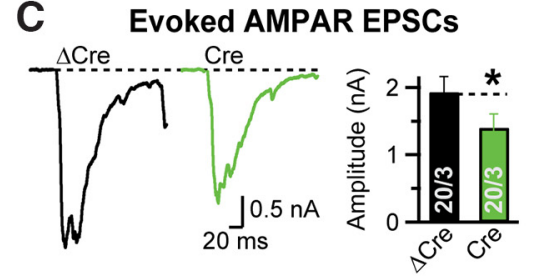

D

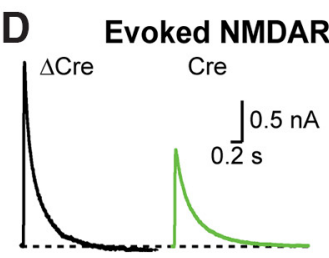

B
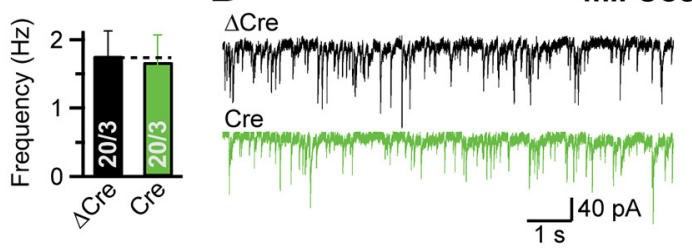

mIPSCs

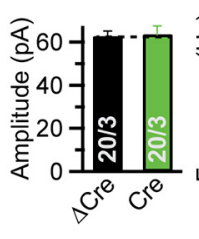

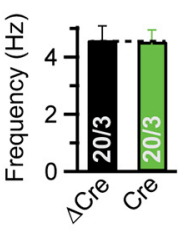

E
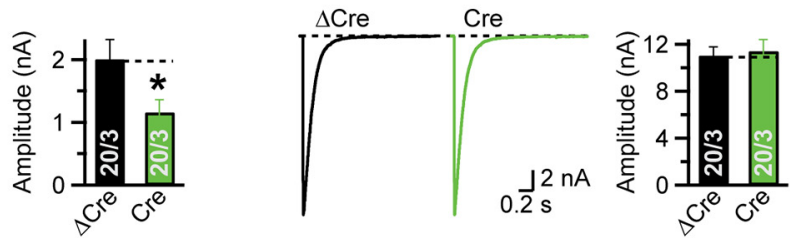

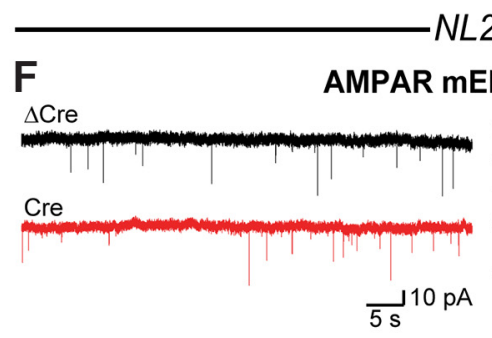

H Evoked AMPAR EPSCs

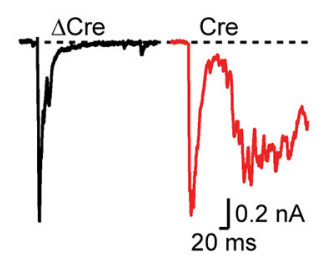

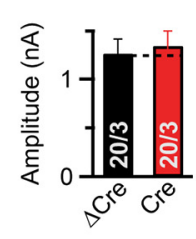

mEPSCs

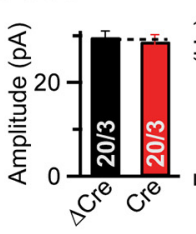

I

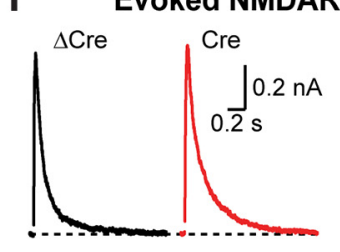

G

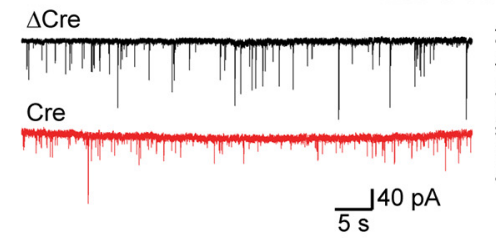

mIPSCs
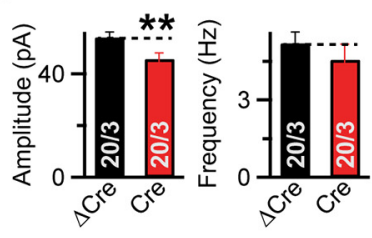

J

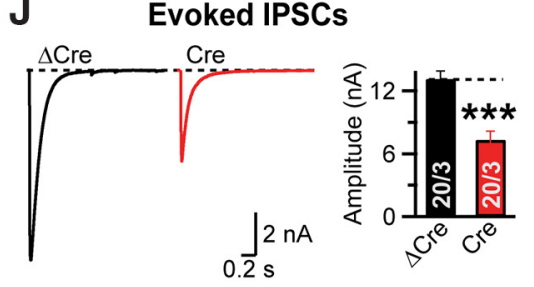

NL3 single cKO (lentivirus-mediated global Cre-infection)

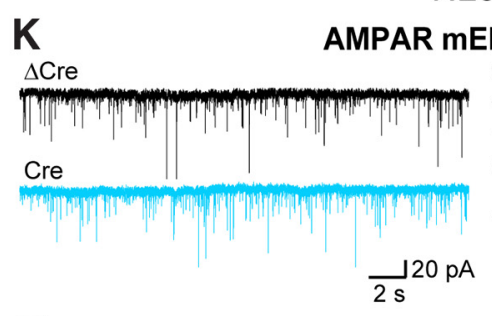

M
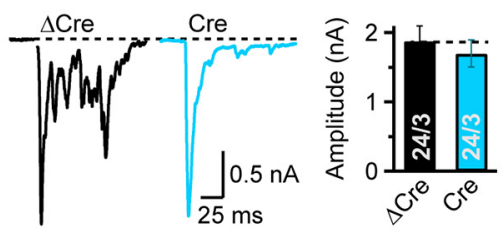

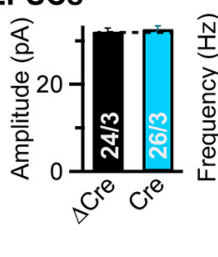

N

N

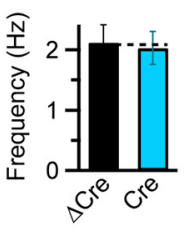

L

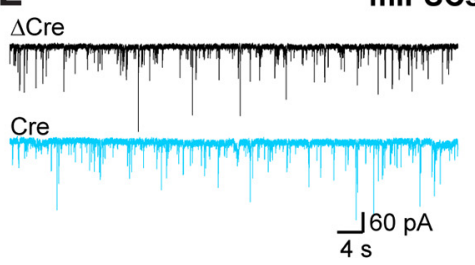

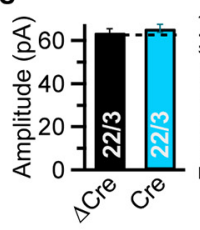

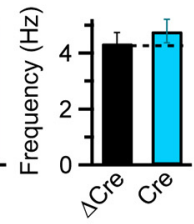

0
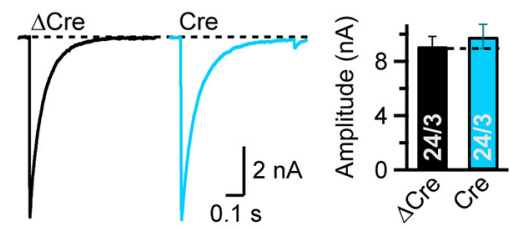

Figure 9. Individual neuroligins perform distinct roles in excitatory and inhibitory synaptic transmission in cultured hippocampal neurons. $A$, Representative traces of AMPAR-mediated mEPSC recordings (left) made from DIV14 to DIV16 primary hippocampal neurons of NL1 single cK0 animals, globally infected with lentivirus expressing $\Delta$ Cre (top) vs Cre-recombinase (bottom) at DIV3. Bar graphs indicate average mEPSC amplitude (left) and frequency (right) for $\Delta$ Cre (black) vs Cre (green) conditions and presented as the mean \pm SEM. $\boldsymbol{B}$, Same as $\boldsymbol{A}$, except for GABA $\mathrm{R}_{\mathrm{A}}-\mathrm{mediated}$ mIPSC recordings. $\boldsymbol{C}-\boldsymbol{E}$, Representative traces of AMPAR-mediated $(\boldsymbol{C})$ and NMDAR-mediated $(\boldsymbol{D})$ evoked EPSCs or GABA $\mathrm{R}_{\mathrm{R}}$-mediated evoked IPSCS $(\boldsymbol{E})$, as recorded from DIV14 to DIV16 primary hippocampal neurons of NL1 cKO animals, globally infected with lentivirus expressing $\Delta$ Cre (black) vs Cre (green) at DIV3. Corresponding bar graphs indicate average amplitudes of evoked EPSCs or IPSCs for $\Delta$ Cre vs Cre condition, and values are represented as the mean \pm SEM. $\boldsymbol{F}-\boldsymbol{J}$, Same as $\boldsymbol{A}-\boldsymbol{E}$, except NL2 single cKO cultures were infected with lentiviruses expressing $\Delta$ Cre (black) vs $\boldsymbol{C r e}$ (red). $\boldsymbol{K}-\mathbf{0}$, Same as $\boldsymbol{A}-\boldsymbol{E}$, except NL3 single cK0 cultures were infected with lentiviruses expressing $\Delta$ (re (black) vs (re (cyan). For individual experimental condition, total number of cells recorded/total number of batches are indicated inside corresponding average bar graphs. Significant differences between $\Delta$ Cre and Cre conditions are indicated with asterisks $\left({ }^{*} p<0.05 ;{ }^{* *} p<0.01 ;{ }^{* *} p<\right.$ 0.005 ; unpaired, one-tailed $t$ test). 


\section{A Neuroligin rescue constructs}

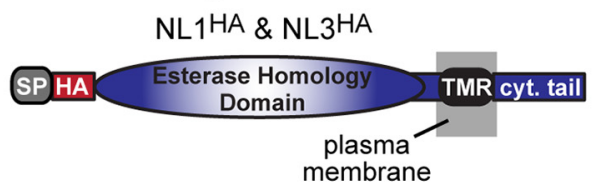

B Experimental design for rescue analyses

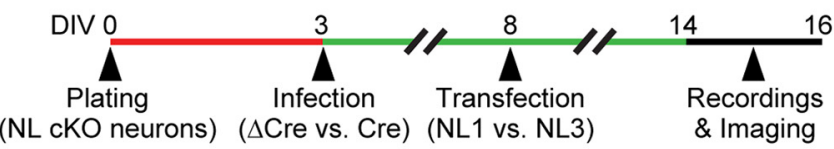

C

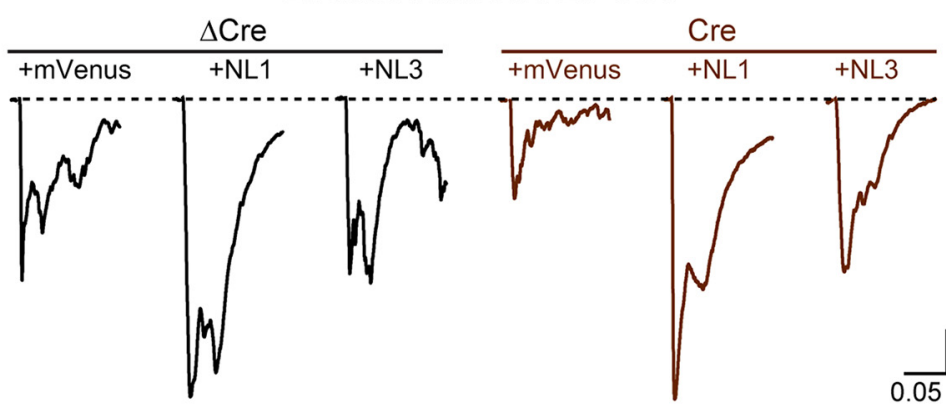

E

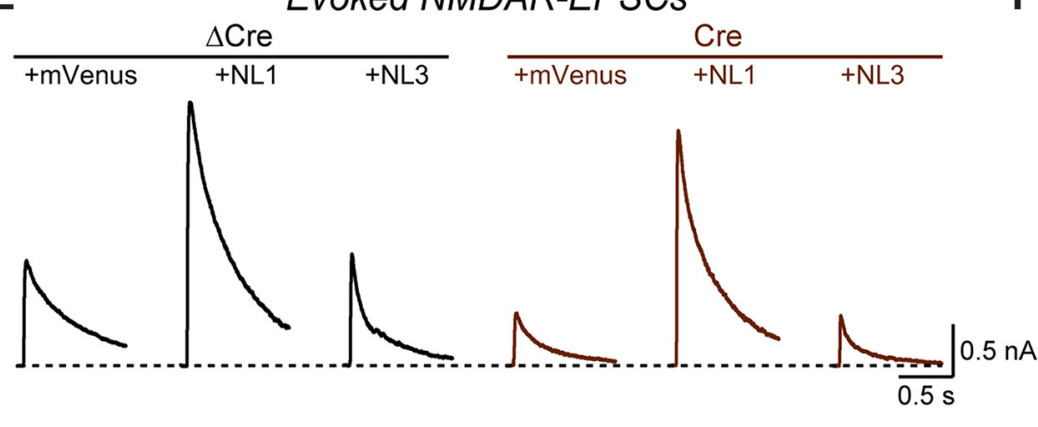

D

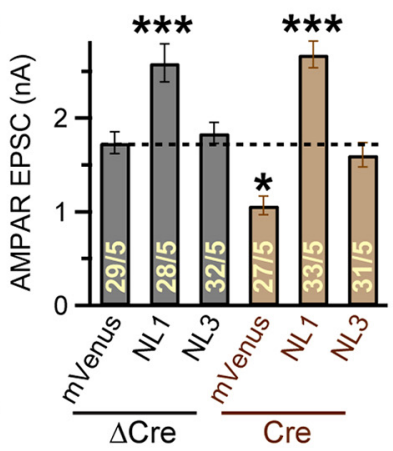

G Spine numbers \& Synapse density

H

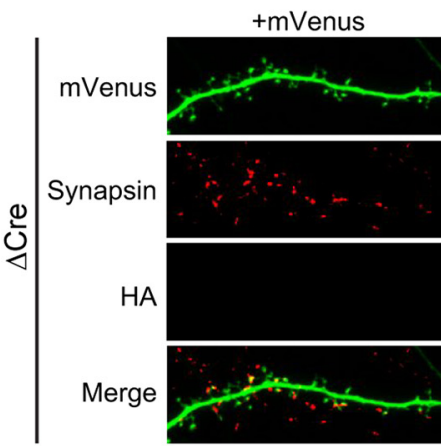

+ mVenus

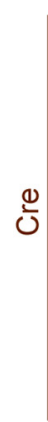
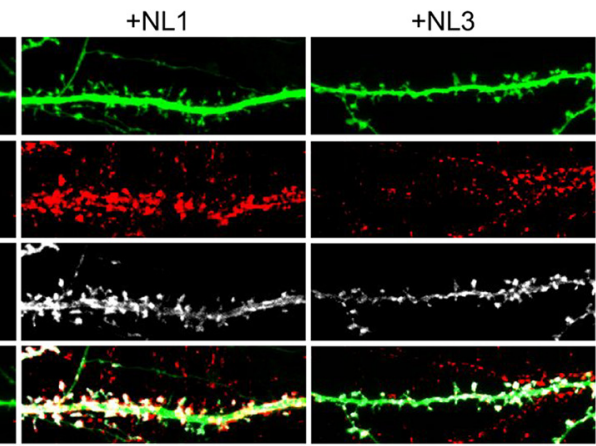

$+\mathrm{NL} 3$

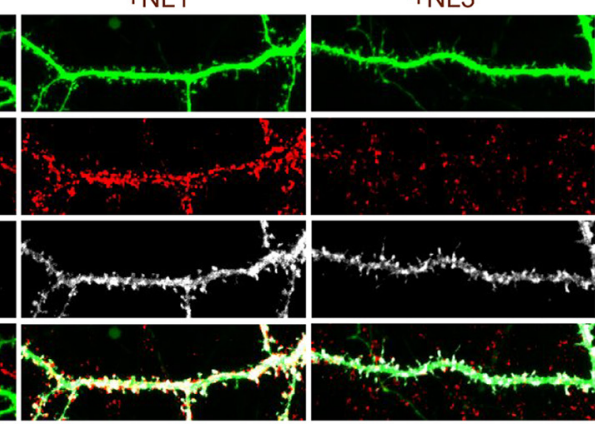

F

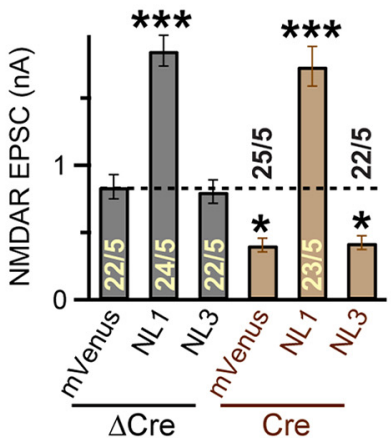

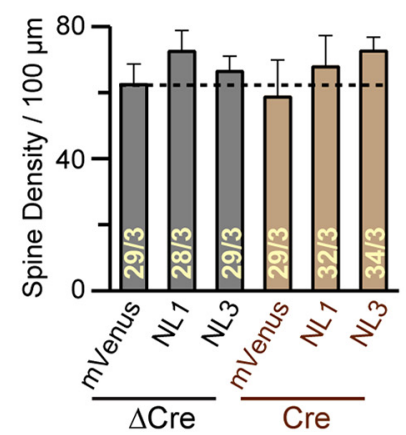

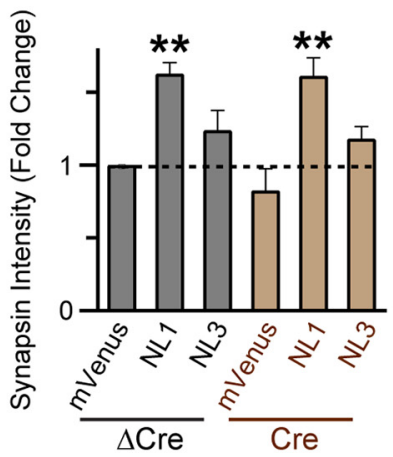

Figure 10. NL1 and NL3 differentially rescue different components of the triple K0 phenotype. $A$, Schematic of domain structures for HA-tagged NL1 and NL3 proteins used, to analyze the effects of neuroligin overexpression in NL123 triple CKO neurons. $\boldsymbol{B}$, Experimental protocol. Hippocampal neurons were cultured from newborn NL123 triple cKO mice, globally infected at DIV3 with lentivirus expressing either $\Delta$ (re or Cre, sparsely cotransfected at DIV8 with plasmids encoding mVenus alone (control) or mVenus together with HA-tagged versions of NL1 or NL3, and analyzed at DIV14-16. C, D, Representative traces $(\boldsymbol{C})$ and average amplitudes $(\boldsymbol{D})$ of AMPAR-mediated evoked EPSCS, recorded from neurons treated with the manipulations described in $\boldsymbol{B}$. $\boldsymbol{E}, \boldsymbol{F}$, Same as $\boldsymbol{C}$ and $\boldsymbol{D}$, but for NMDAR-mediated evoked EPSCs. G, Sample images of dendrites from neurons that were treated with the manipulations described in $\boldsymbol{B}$, (Figure legend continues.) 
A Synaptogenesis time course in cultured cortical neurons

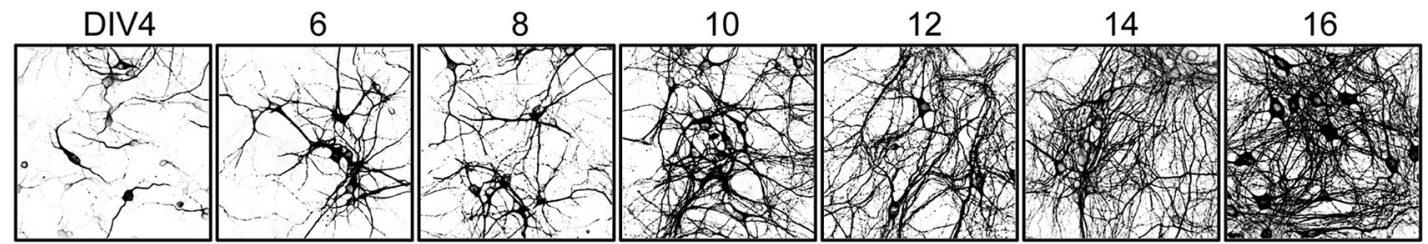

B

B
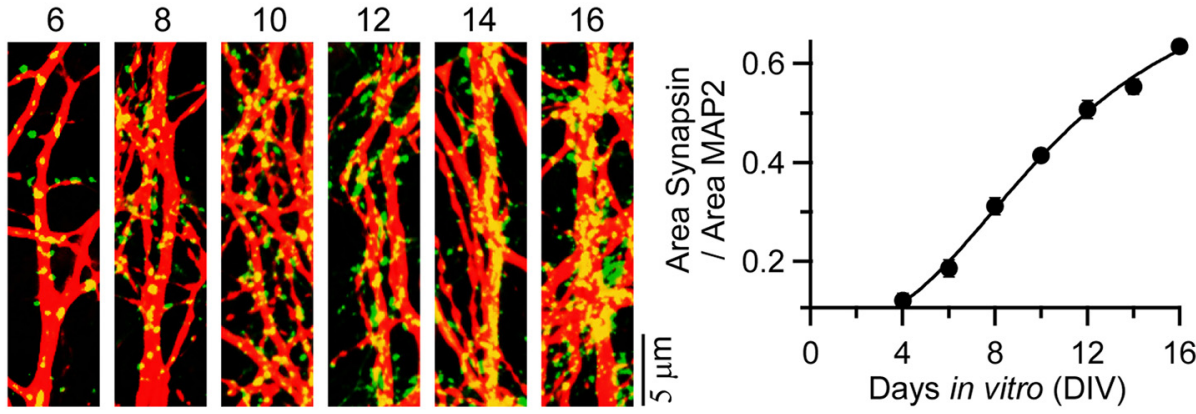

C Lentivirus-mediated global infection efficiency of cortical neurons (DIV 14-16)
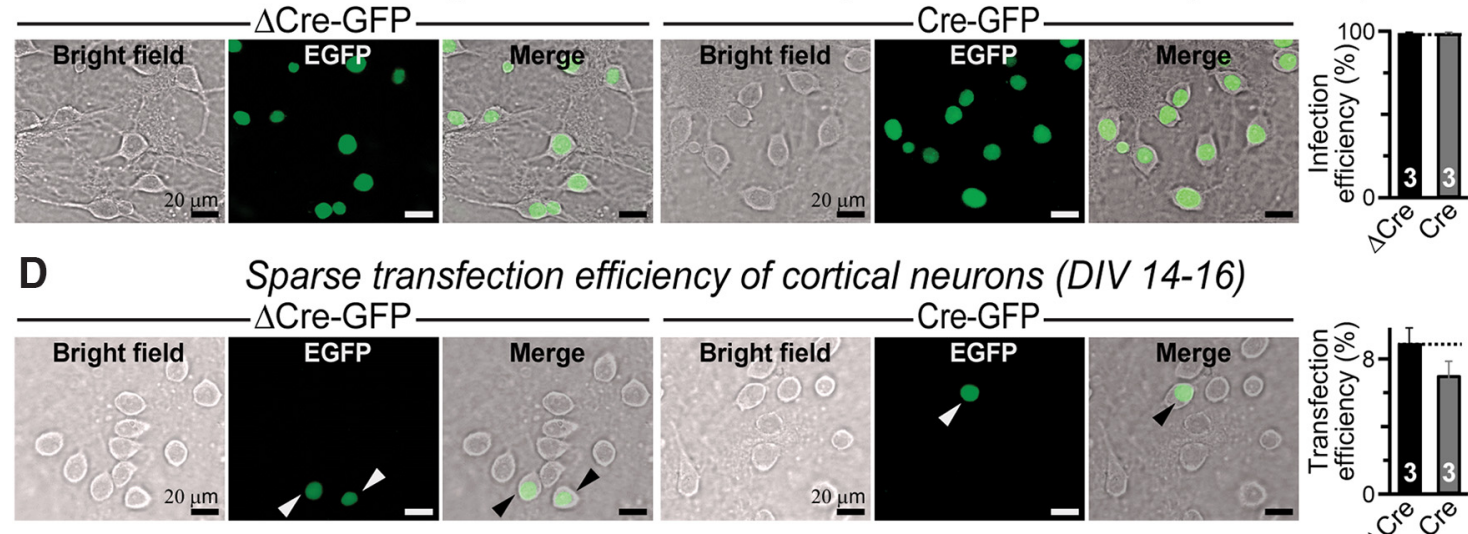

(DIV 14-16)
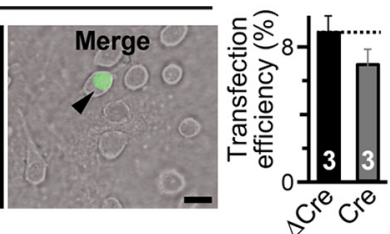

Figure 11. Global vs sparse conditional deletion of NL123 during synaptic development of cultured cortical neurons. $\boldsymbol{A}, \boldsymbol{B}$, Sample images $(\boldsymbol{A})$ demonstrating gradual development of neurite arborization from DIV4 to DIV16 (left to right) in primary cortical culture and representative dendritic sections ( $\boldsymbol{B}$ ) of cortical neurons from DIV4 to DIV16, when coimmunostained for Map2 (red signal) and Synapsin (green signal), indicate gradual increase in synaptic specifications, as quantified (bottom right) by the average values ( $n=20 / \mathrm{DIV}$, mean \pm SEM) of Synapsin area/Map2 area. Data are fit using Hill equation (line) with Hill parameters $P_{\max }=0.81 \pm 0.13, n=2.7 \pm 0.77$, and $K_{1 / 2}=10.53 \pm 1.12 \mathrm{~d}$. C, Representative images (left) of primary cortical neurons derived from NL123 triple cK0 animals, when infected at DIV3 with lentiviruses expressing $\Delta$ Cre-EGFP or Cre-EGFP, and analyzed at DIV14-16 for infection efficiency (right, mean \pm SEM, $n=3$ independent cultures), using nuclear EGFP signal. D, Same as $C$, except for $\mathrm{Ca}^{2+}$ phosphate-mediated sparse transfection (arrowheads) of cultured cortical neurons.

presynaptic terminals of $\Delta$ Cre versus Cre-expressing NL123 triple cKO neurons, when loaded with FM1-43 dye to directly monitor activity-dependent exocytosis (Klingauf et al., 1998), showed very similar destaining kinetics upon depolarization, suggesting that the loss of neuroligins does not impair overall presynaptic release mechanisms (Fig. 6C,D).

To further probe for changes in presynaptic functions in NL123 triple cKO neurons at excitatory versus inhibitory synapses using independent approaches, we next analyzed the CV

$\leftarrow$

(Figure legend continued.) and analyzed by imaging for the transfected mVenus and for immunostained HA neuroligins and synapsin (top panels, $\Delta$ Cre control samples; bottom panels, Cre samples); merged views of all signals are shown at the bottom of each panel. $\boldsymbol{H}$, Summary graphs of spine numbers (top) and synaptic puncta (bottom) as a function of neuroligin overexpression in transfected NL123 triple cKO neurons expressing $\Delta$ Cre (black) vs Cre (brown), as quantified by mVenus-fluorescence or Synapsin staining, respectively. All summary graphs show the mean \pm SEMs; numbers in bars indicate the number of cells patched (for electrophysiology) or dendritic sections analyzed (for imaging)/independent cultures. Statistical comparisons were made by one-way ANOVA with post hoc Tukey's test $\left({ }^{*} p<0.05 ;{ }^{* *} p<0.01 ;{ }^{* * *} p<0.005\right)$. for AMPAR-mediated evoked EPSCs, calculated the PPRs for NMDAR-mediated EPSCs or GABA $\mathrm{A}_{\mathrm{A}}$-mediated IPSCs (note that PPRs for AMPAR could not be determined directly, owing to the network activity that is induced by the first stimulus under AMPAR recording conditions) and assessed the MK801-mediated use-dependent block of NMDAR EPSCs, all of which provide measures of presynaptic release probabilities (Bekkers and Stevens, 1990; Malinow and Tsien, 1990; Rosenmund et al., 1993; Manabe and Nicoll, 1994; Debanne et al., 1996; Raffaelli et al., 2004). We did not detect any significant effect of the neuroligin-1/2/3 triple $\mathrm{KO}$ on these three independent measures of presynaptic release probability, effectively ruling out an effect of postsynaptic neuroligin deletions on presynaptic neurotransmitter release (Fig. 6E-I). Finally, similar to lentivirusmediated global deletion of neuroligins, transfection-based sparse conditional triple KOs of neuroligins also did not alter the CV of AMPAR EPSCs or the PPR of NMDAR EPSCs or $\mathrm{GABA}_{\mathrm{A}} \mathrm{R}$ IPSCs (Fig. 6J-L). These data confirm that postsynaptic loss of neuroligins does not affect the probabilities of release at 


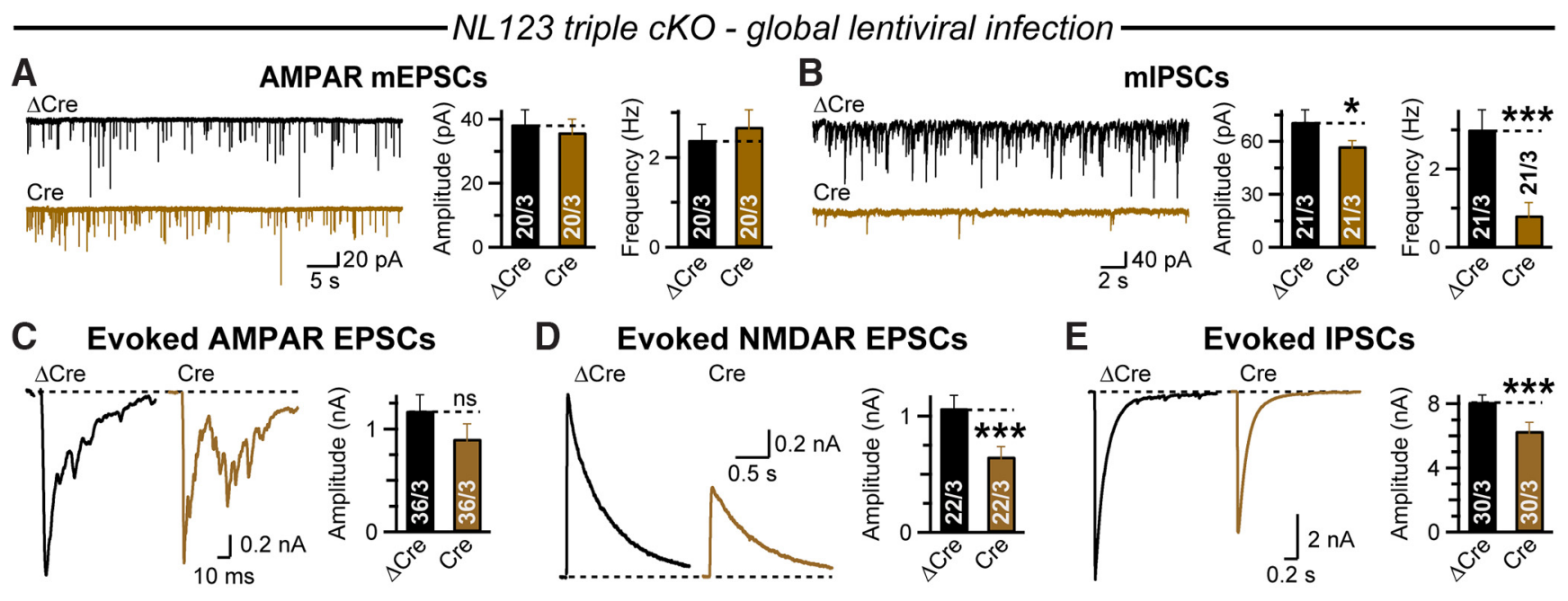

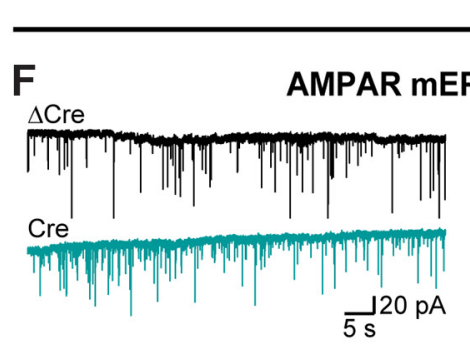

H Evoked AMPAR EPSCs

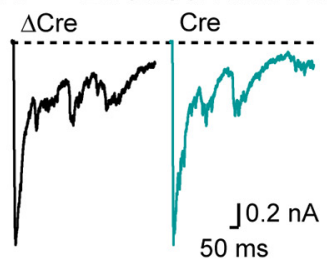

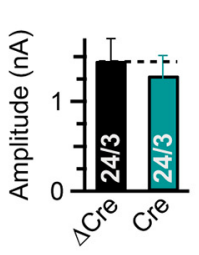

NL123 triple cKO - sparse transfection

G mIPSCs

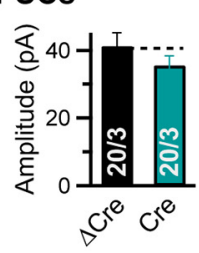

I

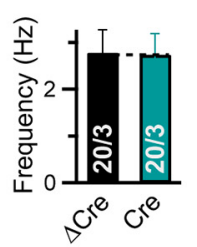

Evol

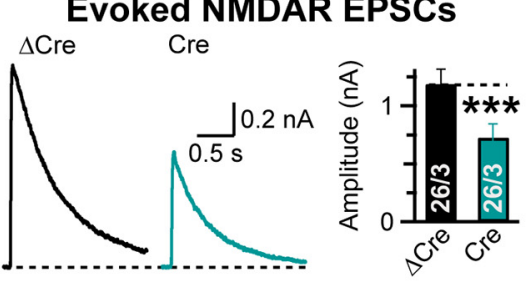

mIPSCs
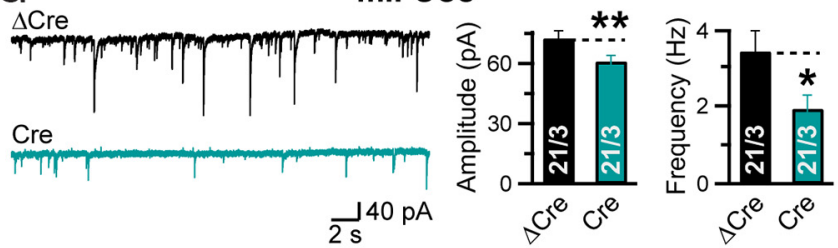

J

Evoked IPSCs

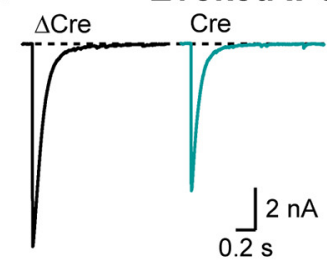

Figure 12. Acute cKO of neuroligins impairs synaptic transmission in cultured cortical neurons similar to hippocampal neurons. $A, B$, Spontaneous mEPSCs $(\boldsymbol{A})$ and mIPSCS $(\boldsymbol{B})$ recorded at DIV14-16 from NL123 triple cK0 primary cortical cultures, which were globally infected with lentivirus expressing $\Delta$ Cre (black) or Cre (brown) at DIV3. Left, Representative traces; right, summary graphs (mean \pm SEM) of the $\mathrm{mEPSC} / \mathrm{mIPSC}$ amplitudes and frequency. C, Representative traces (left) and average peak amplitudes (right, mean \pm SEM) of AMPAR-mediated evoked EPSCS recorded from DIV14-16 cortical neurons expressing $\Delta$ Cre (black) or (re (brown), through lentivirus-mediated global infection at DIV3. D, E, Same as C, except for NMDAR-mediated evoked EPSCs (D) or GABA $A_{A} R$-mediated evoked IPSCS (E). F-J, Same as $\boldsymbol{A}-\boldsymbol{E}$, except for calcium phosphate-mediated sparse transfection ( $\Delta$ Cre in black, and Cre in cyan) at DIV3. For all experiments, total number of cells recorded/independent batches is indicated on bar graphs. Statistical significance between $\Delta$ Cre vs Cre conditions were assessed using unpaired, one-tailed $t$ test (ns, $p>0.05 ;{ }^{*} p<0.05 ;{ }^{* *} p<0.01$; $\left.{ }^{* * *} p<0.005\right)$.

either excitatory or inhibitory synapses in primary hippocampal neurons.

\section{NL123 deletion causes decrease in postsynaptic receptor levels}

Viewed together, the data up to this point suggest that conditional NL123 triple deletions strongly impair synaptic transmission via a postsynaptic mechanism that, based on the decline in mEPSC and mIPSC amplitudes, is mediated at least in part by a decrease in postsynaptic receptor levels. To directly test this hypothesis, we measured the postsynaptic levels of the AMPAR subunit GluA2 by immunocytochemistry of cultured control and NL123-deficient neurons (Fig. $7 A, B$ ). We immunostained surface AMPARs in nonpermeabilized neurons using antibodies to GluA2 followed by permeabilization and immunostaining with antibodies to the presynaptic excitatory synapse marker vGLUT1 (Fig. 7A). Interestingly, we found that the NL123 deletion significantly decreased surface GluA2 puncta size but not density, suggesting a decrease in postsynaptic AMPAR levels consistent with the decrease in mEPSC amplitude (Fig. 7B). Moreover, we de- tected in NL123-deficient neurons a substantial reduction in GluA2 colocalization with vGLUT1, suggesting a loss of AMPARs from some excitatory synapses (Fig. $7 B$ ), which might account for the decrease in mEPSC frequency observed in electrophysiology experiments (see Fig. 3). Thus, endogenous neuroligins support normal postsynaptic localization of AMPARs in hippocampal neurons.

We wondered whether the loss of postsynaptic neuroligins induces a general loss in the surface expression of neurotransmitter receptors. We addressed this question by puffing exogenous AMPA (Clements et al., 1992; Wadiche and Jahr, 2001; Foster et al., 2002; Wong et al., 2003; Crowley et al., 2007; Chanda and Xu-Friedman, 2010 ; in the presence of $\gamma$ DGG, to prevent AMPARs from both desensitization and saturation) or GABA onto the dendritic branches at different positions of a patched neuron and quantifying the resulting currents (Fig. 7C, illustration). Interestingly, we detected significant reductions in the total charge transfers but not in the peak amplitudes of both AMPAR- and $\mathrm{GABA}_{\mathrm{A}} \mathrm{R}$-mediated postsynaptic currents for NL123-deficient neurons, particularly at high agonist concentrations (Fig. $7 D, E$ ). Together, these data 

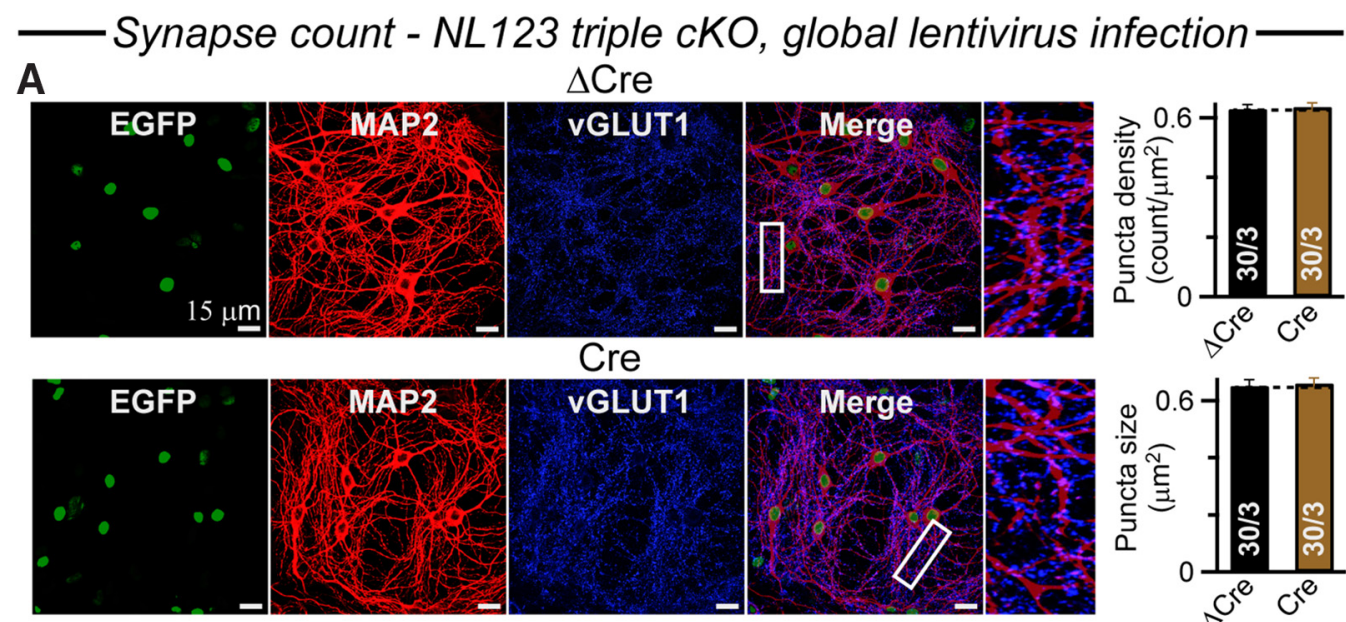

VGLUT1
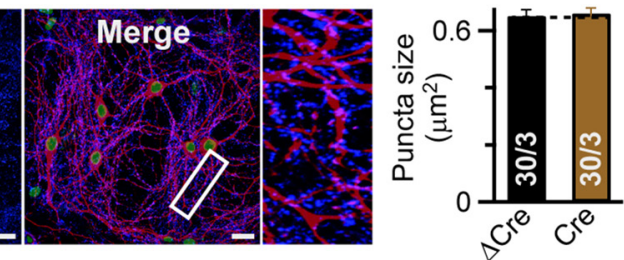

B
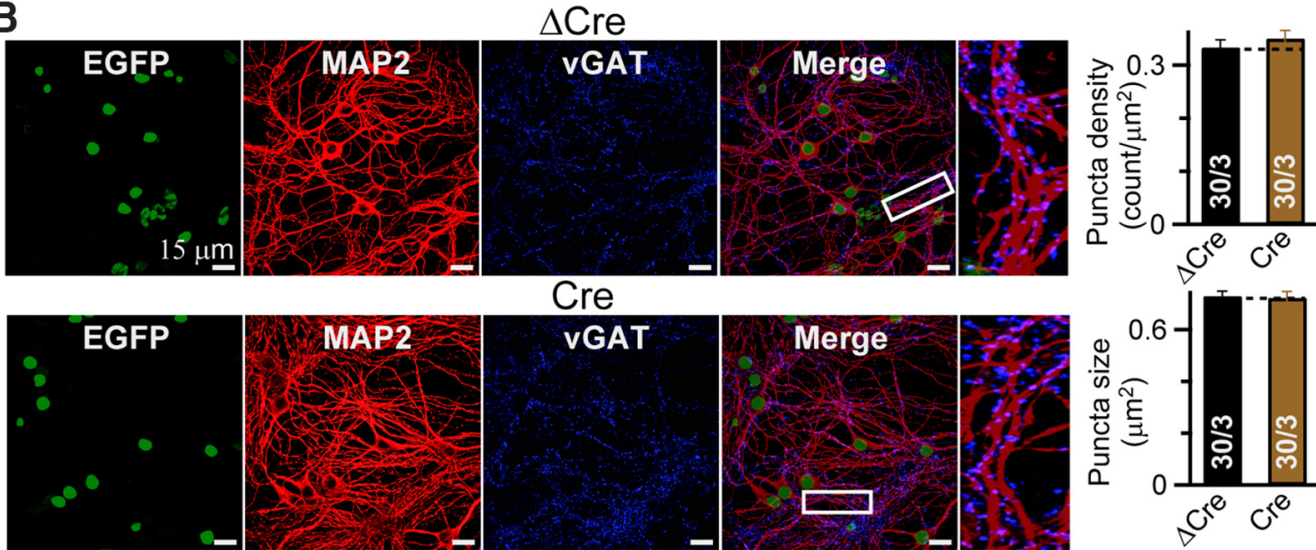

Cre

C

Experimental procedure for spine counting
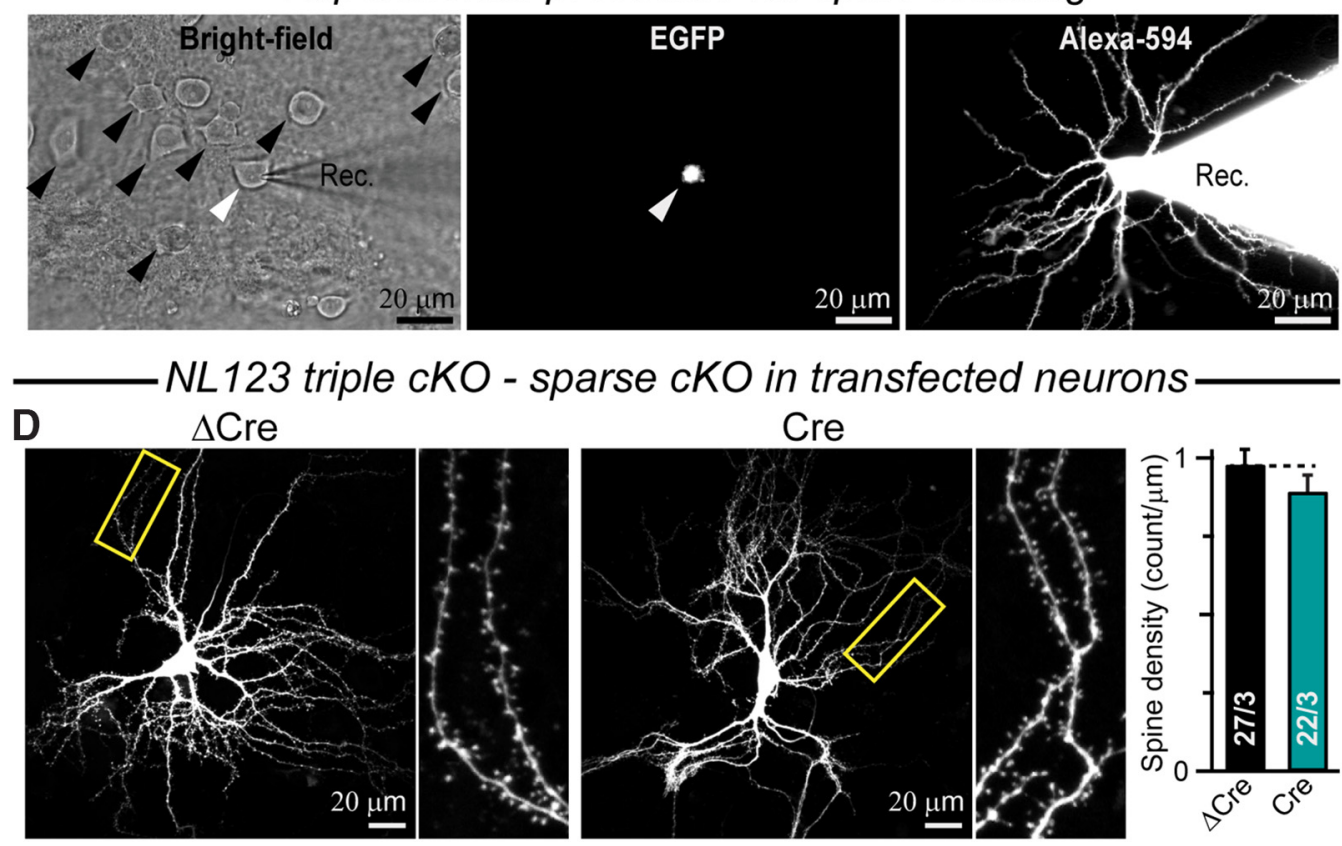

Figure 13. Acute NL123 CKO in cultured cortical neurons does not impair synaptogenesis. A, Conditional deletion of neuroligins does not alter excitatory synapse size and density in cortical neurons cultured from NL123 triple cK0 mice (left, representative images; right, quantifications of synaptic puncta). Neurons were infected with lentiviruses expressing EGFP-fused $\Delta$ Cre (top images) or (re (bottom images) and immunostained for EGFP, MAP2, and vGLUT1 as indicated ("merged" images show the superimposed EGFP, MAP2, and vGlut1 immunofluorescence signals). The boxed area in the merged representative image is expanded in the right image. Summary graphs show average vGLUT1-positive synaptic puncta density (top) or size (bottom). $\boldsymbol{B}$, Same as $\boldsymbol{A}$, except for inhibitory synapses with neurons that were stained for vGAT instead of VGLUT1. C, Experimental strategy to quantify spine density of cortical neurons cultured from NL123 triple cK0 mice, which were sparsely transfected at DIV3 with plasmids expressing $\Delta$ Cre or Cre, and patched at DIV14-16 with a recording pipette (Rec.) containing the fluorescent dye Alexa Fluor-594. Left, Bright-field image (white and black arrowheads indicate transfected and nontransfected cells, respectively). Center, EGFP fluorescence of transfected cells expressing nuclear Cre- or $\Delta$ Cre-EGFP fusion proteins. Right, Alexa Fluor-594 fluorescence of a patched transfected cell. D, Conditional deletion of neuroligins does not alter postsynaptic spine density of cortical (Figure legend continues.) 


\section{A Lentiviral NL123 triple cKO in cultured cortical neurons (DIV28)} $\Delta$ Cre

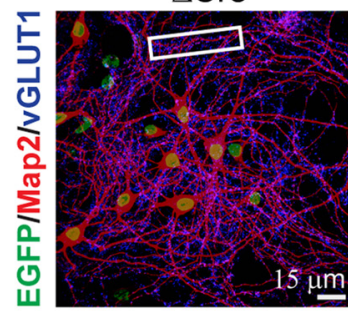

B

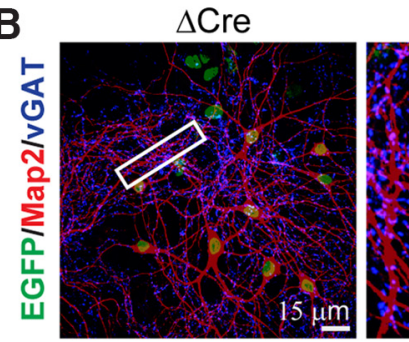
Cre

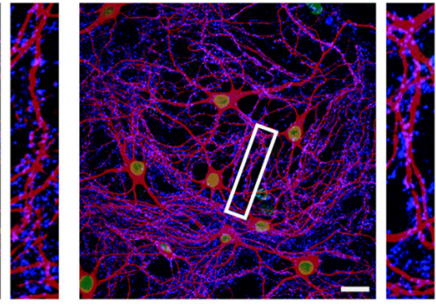

Cre

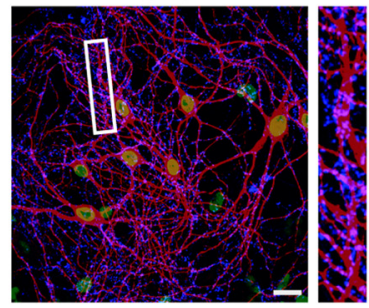

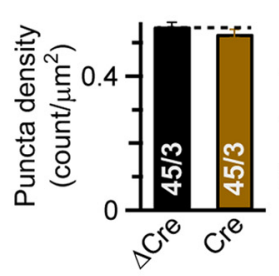
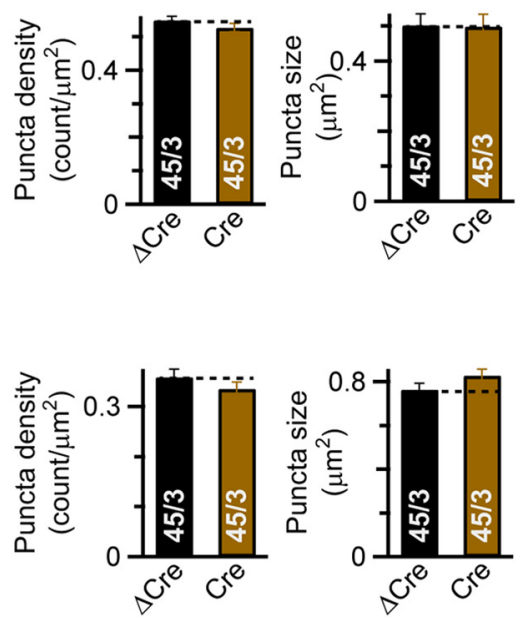

Figure 14. Long-term absence of neuroligins causes no change in synapse numbers for cultured cortical neurons. $\boldsymbol{A}$, Sample images (left) with corresponding boxed areas (white) enlarged for better visualization, and summary graphs (right) representing the density and size of synaptic puncta of cortical neurons from NL123 triple cK0 mice, when infected at DIV3 with lentiviruses expressing $\Delta$ Cre (control) vs (re, and immunostained at DIV28 for vGLUT1 (to label excitatory synapses), MAP2 (to visualize dendrites), and EGFP (to assess infection efficiency since the lentiviruses express (re or $\Delta$ (re as EGFP-fusion proteins). $\boldsymbol{B}$, Same as $\boldsymbol{A}$, except for neurons stained for the inhibitory synapse marker vGAT instead of the excitatory synapse marker vGLUT1. Data are the mean \pm SEM; total number of imaging fields/independent experiments is indicated with corresponding average values. Statistical significance was tested using unpaired, one-tailed $t$ test (for all comparisons, $p>0.05)$.

indicate that neuroligins play an important role in maintaining the synaptic concentration of neurotransmitter receptors and also likely contribute to their membrane trafficking and/or surface retention.

\section{Neuroligin isoforms differentially contribute to excitatory and inhibitory synapse function}

Next, we aimed to segregate the specific functional contributions of various neuroligin isoforms to the synaptic phenotypes observed in NL123-deficient neurons. We first analyzed NL13 or NL23 double deletions using hippocampal neurons cultured from the respective double cKO mice and infected them with $\Delta$ Cre-EGFP- or Cre-EGFP-expressing lentiviruses (Fig. 8). We found that the NL13 double deletion caused selective changes in excitatory synaptic transmission, with a small impairment in the mEPSC amplitude but not frequency (Fig. $8 A, \sim 10 \%$ ), no changes in mIPSC amplitude and frequency (Fig. $8 B$ ), a significant decline in AMPAR and NMDAR EPSCs (Fig. $8 C, \sim 35 \%$ decrease, $D$, $\sim 50 \%$ decrease), and no change in $\mathrm{GABA}_{\mathrm{A}} \mathrm{R}$-evoked IPSCs (Fig. $8 E$ ). In contrast, the NL23 double deletion induced a selective, although modest, decrease in inhibitory synaptic transmission, with no changes in mEPSCs (Fig. $8 F$ ), a robust decrease in mIPSC amplitude but not frequency (Fig. $8 G, \sim 25 \%$ ), no changes in AMPAR and NMDAR EPSCs (Fig. $8 H, I$ ), and a significant decrease in $\mathrm{GABA}_{\mathrm{A}} \mathrm{R}$-mediated evoked IPSCs (Fig. 8J, $\sim 20 \%$ decrease).

(Figure legend continued.) neurons, as measured with Alexa Fluor-594 fluorescence. Left and center, Sample images of Alexa Fluor-594-infused neurons (boxed dendritic sections are expanded on the right for each cell), sparsely transfected with $\Delta$ (re vs (re-expressing vectors. Right, Summary graphs depict spine densities from corresponding conditions. Bar graphs show mean $\pm S E M$, with the number of imaging fields (for global manipulations) or neurons (for sparse manipulations)/independent cultures analyzed depicted in the bars. No significant differences ( $p>0.05$, unpaired, one-tailed $t$ test) were observed between $\Delta$ Cre vs Cre conditions for any of the quantifications shown.
To further dissect the contributions of individual neuroligins, we systematically analyzed NL1, NL2, and NL3 cKO mice using Cre-mediated global deletions in hippocampal neurons cultured from the respective single $\mathrm{cKO}$ mice (Fig. 9). The results were straightforward: the NL1 deletion impaired only evoked AMPARand NMDAR-mediated EPSCs (Fig. $9 A-E, ~ 30 \%$ and $45 \%$ decreases, respectively), whereas the NL2 deletion impaired only the amplitude of mIPSCs and GABAR-mediated IPSCs (Fig. $9 F-J, \sim 10 \%$ and $50 \%$ decreases, respectively). The NL3 deletion, in contrast, on its own caused no significant change (Fig. 9K-O). In aggregate, these data suggest that NL1 and NL2 perform nonredundant functions at excitatory and inhibitory synapses, respectively, and that their loss-of-function phenotypes in triple NL123-deficient neurons is amplified by additional deletion of NL3, which by itself has no significant essential effect.

\section{Comparative analysis of the excitatory synaptic effects generated by NL1 versus NL3 overexpressions}

The single, double, and triple neuroligin $\mathrm{KO}$ analyses suggest that neuroligin isoforms act in a nonredundant fashion to establish synaptic strength but do not contribute to synapse formation. To corroborate this conclusion, we performed "rescue" experiments in which we tested the effects of NL1 and NL3 overexpression on synaptic function and of synapse and spine numbers on control and NL123-deficient cultured hippocampal neurons (Fig. 10). We infected hippocampal neurons cultured from NL123 triple cKO mice at DIV3 with lentiviruses encoding $\Delta$ Cre-EGFP or Cre-EGFP and sparsely transfected them at DIV8 with mVenus alone (for visualization of transfected cells) or with mVenus and also either HA-tagged NL1 or NL3. We then analyzed the neurons at DIV14-16 using electrophysiology and morphological assays (Fig. 10A, B).

Consistent with earlier results (Chubykin et al., 2007; Ko et al., 2009a), we observed that in control neurons expressing $\Delta$ CreEGFP, NL1 overexpression caused a dramatic increase in both AMPAR- and NMDAR-mediated evoked EPSC amplitudes (Fig. 
$10 C-F)$. This effect was much larger for NMDAR EPSCs $(\sim 200 \%)$ than for AMPAR EPSCs $(\sim 50 \%)$. NL3 overexpression, conversely, had no effect on either synaptic response. In parallel experiments, NL123-deficient neurons expressing Cre-EGFP showed significant decrease in AMPAR and NMDAR EPSC amplitudes as expected (Fig. 10C-F), as before (see Fig. 3); this decrease was more severe for NMDAR EPSCs $(\sim 50 \%)$ than for AMPAR EPSCs $(\sim 30 \%)$. NL1 overexpression (rescue) in NL123deficient neurons not only reversed this deficit, but enhanced AMPAR and NMDAR EPSCs far above control levels in a manner similar to that in NL1 overexpression in control neurons. Intriguingly, NL3 overexpression reversed the AMPAR-EPSC deficit to control levels, but had no effect on the NMDAR-EPSC deficit (Fig. 10C-F). Thus, functionally, NL1 and NL3 behave very differently not only in terms of their KO phenotype, but also for their dominant-positive effects when overexpressed.

We next examined in the same neurons the effects of the NL1 and NL3 overexpression on dendritic spine and synapse numbers. Using immunostaining with HA antibody, we detected strong expression of the transfected NL1 and NL3 on dendrites and spines that often coincided with synapsin staining as a marker for synapses (Fig. 10G). We quantified the density of dendritic spines that were identified based on the mVenus signal, and of synapses using the intensity of synapsin staining as a proxy (Fig. $10 H)$. None of the various manipulations had any effect on spine numbers, even though overexpression of NL1, but not of NL3, caused a dramatic increase $(\sim 60 \%)$ in synapsin staining (Fig. $10 \mathrm{H}$ ). Viewed together, these data indicate that overexpression of NL1, but not of NL3, causes increased production of synapses, but not of spines, and that the relatively greater functional requirement for NL1 in NMDAR-mediated than in AMPAR-mediated synaptic responses is replicated in the properties of these newly induced synapses. These data corroborate the notion that NL1 and NL3 have intrinsically different functions despite their homology and common targeting to excitatory synapses.

\section{Neuroligin function in cortical neurons mimics their role in hippocampal neurons}

To assess potential region-specific effects of neuroligins (Etherton et al., 2011) and to examine the generality of the results described above, we tested the role of neuroligins in neurons cultured from cortex instead of hippocampus. In cultured cortical neurons, the synapse density increased gradually from DIV4 to DIV16 (Fig. 11A), which is similar to our observations in cultured hippocampal neurons (see Fig. 1). Moreover, global and sparse deletions of neuroligins in cortical neurons by lentiviral infection and transfection, respectively, were similarly efficient to those in hippocampal neurons (Fig. 11C,D) and, thus, afforded another ready preparation for studying endogenous neuroligin function.

We found that the NL123 triple KO caused impairments in synaptic transmissions in cortical neurons similar to those observed in hippocampal neurons, although an AMPAR-specific phenotype was not detected in cortical cultures, and the NMDAR-/ $\mathrm{GABA}_{\mathrm{A}} \mathrm{R}$-mediated synaptic phenotypes were less severe in cortical than in hippocampal neurons (Fig. 12). Again, essentially the same phenotype was observed after global or sparse neuroligins deletions. Moreover, as in hippocampal neurons, we observed that the NL123 triple deletion had no effect on synapse density or spine numbers (Fig. 13). Even after an extended culture period of 4 weeks, no decrease in the density or size of synaptic puncta was detected in NL123-deficient cortical neurons (Fig. 14). Thus, conditional deletion of endogenous neuroligins does not affect overall synaptogenesis and/or spinogenesis in cultured cortical neurons, corroborating the data from cultured hippocampal neurons.

\section{Discussion}

Increasing evidence suggests that presynaptic neurexins and their multifarious trans-synaptic ligands are central organizers of synapses (Südhof, 2008; Krueger et al., 2012). The relative binding affinities of various neurexin ligands, including those of neuroligins, leucine-rich repeat transmembrane neuronal proteins (LRRTMs), neurexophilins, and cerebellins, depend on the specific neurexin and ligand isoforms involved and on the alternative splicing of these isoforms. Moreover, some of the neurexin ligands compete with each other for binding, adding a higher layer of complexity (Ichtchenko et al., 1995; Missler et al., 1998; Boucard et al., 2005; Chih et al., 2006; Ko et al., 2009a,b; de Wit et al., 2009; Siddiqui et al., 2010; Matsuda and Yuzaki, 2011). Thus, neurexins and their ligands form a dynamic trans-synaptic interaction network that is regulated by the expression and alternative splicing of neurexins and of their various binding partners. Strikingly, all neurexin ligands appear to be expressed in multiple isoforms, but it remains unclear whether these isoforms are functionally redundant or perform distinct and diverse roles.

Among neurexin ligands, neuroligins are possibly the most important because they, together with $\alpha$-neurexins, appear to be conserved in invertebrates (Tabuchi and Südhof, 2002; Li et al., 2007; Banovic et al., 2010; Chen et al., 2010; Sun et al., 2011; Owald et al., 2012; Xing et al., 2014). Despite considerable efforts, the cellular functions and molecular mechanisms of neuroligins are incompletely understood. Two key questions in particular appear to be important. First, neuroligin overexpression causes large increases in synaptogenesis, and KDs, using shRNAs or microRNAs, generally induce dramatic losses of synapses, but genetic deletions mostly induce impairments in synaptic function with no or only minor changes in synapse numbers. Thus, different approaches lead to different conclusions about neuroligin function. Second, vertebrates express multiple neuroligin genes that bind to neurexins by similar mechanisms, but it is unclear whether these genes perform similar or distinct functions. The high degree of homology between neuroligin isoforms suggests similar biological activities, yet their distinct localizations and different synapse specificities indicate divergent functions.

In the present study, we set out to systematically approach these questions with two principal objectives: to produce a comprehensive characterization of the general cellular function of neuroligins; and to gain insight into their isoform-specific mechanistic contributions. For the first aim, we acutely deleted all major neuroligin isoforms using NL123 triple cKO in cultured hippocampal and cortical neurons; for the second aim, we removed neuroligin isoforms, in combination or individually, and additionally performed overexpression experiments of specific neuroligins. For both objectives, we analyzed the effects of the molecular manipulations on synapse formation and function using imaging and electrophysiology. To the best of our knowledge, this is the first systematic analysis of neuroligin function using such complementary approaches in cultured neurons. We believe that this analysis enabled several fundamental conclusions about the role of neuroligins that are relevant for the two key questions raised above.

First, we observed that the deletion of neuroligins impaired the strength of basal synaptic transmission in both cultured hippocampal and cortical neurons (Figs. 3, 12), indicating that neu- 
roligins play essential roles in synapse function. However, the loss of neuroligins did not induce a major decrease in synaptogenesis, independent of culture durations or whether the neuroligins were deleted using global or sparse manipulations (Figs. 4, 5, 13, 14). These results complement previous studies using constitutive KOs in cultured neurons (Varoqueaux et al., 2004; Chubykin et al., 2007; Blundell et al., 2010) and conditional KOs in vivo (Liang et al., 2015; Zhang et al., 2015) to establish that neuroligins by themselves are not essential for synapse formation. These results are at odds, however, with various KD studies (Chih et al., 2005; Shipman et al., 2011; Kwon et al., 2012). We argue that genetic manipulations are more interpretable than KD approaches, especially if the genetic manipulations are performed using conditional KOs that operate on a time frame similar to that of $\mathrm{KD}$ approaches. KD approaches invariably adopt the endogenous microRNA-based regulatory machinery of a cell; thus, even if an shRNA or microRNA does not have a specific off-target effect, such approaches inevitably change the endogenous regulatory microRNA machinery and are thus likely to cause general changes in neurons (Bacaj et al., 2015). Of note, it has been reported that a complete block of postsynaptic receptors by selective pharmacological agents causes severe defects in spine morphology (McKinney et al., 1999). In our present study, the NL123 triple KO neurons displayed a significant but partial silencing of excitatory synapses, as is evident from electrophysiology and imaging experiments (Figs. 3, 7, and 12). Although it is possible that the extent and/or the duration of synapse silencing may differentially affect spine formation and/or maintenance, pharmacological inhibitions can also have secondary effects due to non-synapse-specific processes.

Second, we observed that the NL123 triple deletion did not significantly alter the presynaptic functional properties of excitatory or inhibitory synapses, arguing against any effects on release probability (Fig. 6). Overexpression of NL1 has been reported to decrease PPRs of AMPAR- and NMDAR-mediated EPSCs in CA1 neurons via a retrograde mechanism (Futai et al., 2007); however, we found no such effect, and neither did a previous study (Soler-Llavina et al., 2011) on NL1 constitutive KOs despite a reduction in NMDA/AMPA ratio. It is possible that the overexpression of exogenous neuroligins may cause gain-offunction effects, which might not reflect the cellular function of endogenous neuroligins.

Third, deletion of endogenous neuroligins decreased the synaptic localization of AMPARs in hippocampal neurons and also moderately reduced their overall surface levels (Fig. 7). A loss of postsynaptic receptor levels as a mechanism of action of neuroligin deletions is supported by the decrease in mini-amplitudes (Fig. 3). Thus, deletions of neuroligins may generally decrease the levels of synaptic receptors in postsynaptic specializations and could even produce synapse silencing.

Fourth, the cellular effects of conditional neuroligin deletions were identical between global deletions in all neurons and sparse deletions in a small subset of neurons (Figs. 3, 4, 12, 13). These results show not only that neuroligins act via a postsynaptic, cellautonomous mechanism, but also that there is no loss of competitiveness of a neuroligin-deficient neuron for synapse formation when it is surrounded by neuroligin-expressing neurons.

Fifth, deletion of individual neuroligin isoforms differentially affected the functional properties of excitatory versus inhibitory synapses (Figs. 8, 9). In hippocampal neurons, NL1 and NL2 deletions decreased the amplitude of NMDAR-mediated excitatory and of $\mathrm{GABA}_{\mathrm{A}} \mathrm{R}$-mediated inhibitory synaptic responses, respectively (Figs. 8, 9), consistent with previous findings (Chubykin et al., 2007; Poulopoulos et al., 2009). Interestingly, acute loss of NL1 alone significantly impaired AMPAR-mediated basal EPSCs especially in hippocampal neurons (Fig. 9C), which was surprising because a number of previous studies based on NL1 constitutive KOs uncovered no such phenotype in CA1 neurons of hippocampal slices (Chubykin et al., 2007; Blundell et al., 2010; Budreck et al., 2013), although NL1 traps AMPAR surface diffusion at postsynaptic specifications (Mondin et al., 2011). This difference could arise from possible compensatory mechanisms in constitutive NL1 KOs. In support of this hypothesis, we found that the AMPAR phenotype was even more pronounced in NL13 double KO cells (Fig. $8 A, C$ ), suggesting that NL3 may play a minor but redundant role at excitatory synapses and that the presence of NL3 in NL1 constitutive KO cells may further mask its AMPAR-specific effects. Thus, although our present study does not distinguish between different cell types in primary cultures, our data suggest that NL1 can regulate AMPAR-mediated synaptic transmission at least in a subset of synapses.

Sixth, overexpression of NL1 alone in control neurons dramatically increased both NMDAR- and AMPAR-mediated excitatory synaptic transmission, which directly correlated with enhanced synaptogenesis (Fig. 10), in accordance with previous studies (Dean et al., 2003; Graf et al., 2004; Prange et al., 2004; Boucard et al., 2005; Chih et al., 2005; Chubykin et al., 2007; Ko et al., 2009a). However, when overexpressed in neuroligin-deficient neurons, NL1 did not simply rescue the corresponding deficiencies in NMDAR- and AMPAR-mediated synaptic currents, but produced the same overexpression phenotype of these currents as in control neurons (Fig. 10C-F). Furthermore, NL1 overexpression in neuroligin-deficient neurons caused the same prominent increase in synaptogenesis as in control neurons, even though the loss of all neuroligin isoforms failed to produce any effect on synaptogenesis (Fig. 10G,H). Thus, overexpressed NL1 can assume gain-of-function effects.

Seventh and finally, in a comparison between NL1 and NL3 overexpressions, we noticed that these two neuroligin isoforms display striking differences in their mechanistic contributions that resemble the differences in their KO phenotypes (Fig. 10). In contrast to NL1, NL3 overexpression in a control background did not affect any form of excitatory synaptic transmissions, suggesting that NL3 may not be essential for excitatory synapse function in the presence of NL1. Interestingly, in NL123 triple cKO neurons overexpressed NL3 alone rescued the impairments in AMPAR-mediated but not NMDAR-mediated EPSCs (Fig. 10C-F). These data suggest that NL3 may directly regulate AMPARs via cellular mechanisms that are different from NL1, which mainly operates through NMDAR-dependent mechanisms (Chubykin et al., 2007). Moreover, unlike NL1, overexpression of NL3 did not cause apparent changes in synapse numbers (Fig. 10G,H). Thus, the effects of neuroligin overexpression on synaptic transmission seem to reflect a physiological activity that indicates a functional diversification of these proteins.

In summary, our data suggest that although neuroligin overexpression phenotypes likely report on a physiological function of neuroligins, they also lead to gain-of-function effects whose interpretation is more difficult, such as the induction of synapses without increasing spine numbers. In contrast, acute genetic lossof-function approaches indicate that different neuroligins perform distinct roles in enabling synaptic transmission at different types of synapses and shaping the properties of these synapses. Based on these data, we propose that neuroligins are part of a general trans-synaptic signaling machinery that organizes syn- 
apses in a manner specified by the complementary expressions of interacting presynaptic and postsynaptic cell adhesion molecules.

\section{References}

Araç D, Boucard AA, Ozkan E, Strop P, Newell E, Südhof TC, Brunger AT (2007) Structures of neuroligin-1 and the neuroligin-1/neurexin-1 beta complex reveal specific protein-protein and protein-Ca2+ interactions. Neuron 56:992-1003. CrossRef Medline

Bacaj T, Ahmad M, Jurado S, Malenka RC, Südhof TC (2015) Synaptic function of Rab11Fip5: selective requirement for hippocampal long-term depression. J Neurosci 35:7460-7474. CrossRef Medline

Banovic D, Khorramshahi O, Owald D, Wichmann C, Riedt T, Fouquet W, Tian R, Sigrist SJ, Aberle H (2010) Drosophila neuroligin 1 promotes growth and postsynaptic differentiation at glutamatergic neuromuscular junctions. Neuron 66:724-738. CrossRef Medline

Bekkers JM, Stevens CF (1990) Presynaptic mechanism for long-term potentiation in the hippocampus. Nature 346:724-729. CrossRef Medline

Blundell J, Blaiss CA, Etherton MR, Espinosa F, Tabuchi K, Walz C, Bolliger MF, Südhof TC, Powell CM (2010) Neuroligin-1 deletion results in impaired spatial memory and increased repetitive behavior. J Neurosci 30 : 2115-2129. CrossRef Medline

Bolliger MF, Pei J, Maxeiner S, Boucard AA, Grishin NV, Südhof TC (2008) Unusually rapid evolution of Neuroligin-4 in mice. Proc Natl Acad Sci U S A 105:6421-6426. CrossRef Medline

Boucard AA, Chubykin AA, Comoletti D, Taylor P, Südhof TC (2005) A splice code for trans-synaptic cell adhesion mediated by binding of neuroligin 1 to alpha- and beta-neurexins. Neuron 48:229-236. CrossRef Medline

Budreck EC, Scheiffele P (2007) Neuroligin-3 is a neuronal adhesion protein at GABAergic and glutamatergic synapses. Eur J Neurosci 26:17381748. CrossRef Medline

Budreck EC, Kwon OB, Jung JH, Baudouin S, Thommen A, Kim HS, Fukazawa Y, Harada H, Tabuchi K, Shigemoto R, Scheiffele P, Kim JH (2013) Neuroligin- 1 controls synaptic abundance of NMDA-type glutamate receptors through extracellular coupling. Proc Natl Acad Sci U S A 110: 725-730. CrossRef Medline

Chanda S, Xu-Friedman MA (2010) A low-affinity antagonist reveals saturation and desensitization in mature synapses in the auditory brain stem. J Neurophysiol 103:1915-1926. CrossRef Medline

Chanda S, Marro S, Wernig M, Südhof TC (2013) Neurons generated by direct conversion of fibroblasts reproduce synaptic phenotype caused by autism-associated neuroligin-3 mutation. Proc Natl Acad Sci U S A 110: 16622-16627. CrossRef Medline

Chanda S, Aoto J, Lee SJ, Wernig M, SüdhofTC (2016) Pathogenic mechanism of an autism-associated neuroligin mutation involves altered AMPAreceptor trafficking. Mol Psychiatry 21:169-177. CrossRef Medline

Chen K, Gracheva EO, Yu SC, Sheng Q, Richmond J, Featherstone DE (2010) Neurexin in embryonic Drosophila neuromuscular junctions. PLoS One 5:e11115. CrossRef Medline

Chen X, Liu H, Shim AH, Focia PJ, He X (2008) Structural basis for synaptic adhesion mediated by neuroligin-neurexin interactions. Nat Struct Mol Biol 15:50-56. CrossRef Medline

Chih B, Engelman H, Scheiffele P (2005) Control of excitatory and inhibitory synapse formation by neuroligins. Science 307:1324-1328. CrossRef Medline

Chih B, Gollan L, Scheiffele P (2006) Alternative splicing controls selective trans-synaptic interactions of the neuroligin-neurexin complex. Neuron 51:171-178. CrossRef Medline

Chubykin AA, Atasoy D, Etherton MR, Brose N, Kavalali ET, Gibson JR, Südhof TC (2007) Activity-dependent validation of excitatory versus inhibitory synapses by neuroligin-1 versus neuroligin-2. Neuron 54:919931. CrossRef Medline

Clements JD, Lester RA, Tong G, Jahr CE, Westbrook GL (1992) The time course of glutamate in the synaptic cleft. Science 258:1498-1501. CrossRef Medline

Comoletti D, Flynn R, Jennings LL, Chubykin A, Matsumura T, Hasegawa H, Südhof TC, Taylor P (2003) Characterization of the interaction of a recombinant soluble neuroligin-1 with neurexin-1beta. J Biol Chem 278: 50497-50505. CrossRef Medline

Comoletti D, Flynn RE, Boucard AA, Demeler B, Schirf V, Shi J, Jennings LL, Newlin HR, Südhof TC, Taylor P (2006) Gene selection, alternative splicing, and post-translational processing regulate neuroligin selectivity for beta-neurexins. Biochemistry 45:12816-12827. CrossRef Medline
Crowley JJ, Carter AG, Regehr WG (2007) Fast vesicle replenishment and rapid recovery from desensitization at a single synaptic release site. J Neurosci 27:5448-5460. CrossRef Medline

Dean C, Scholl FG, Choih J, DeMaria S, Berger J, Isacoff E, Scheiffele P (2003) Neurexin mediates the assembly of presynaptic terminals. Nat Neurosci 6:708-716. CrossRef Medline

Debanne D, Guérineau NC, Gähwiler BH, Thompson SM (1996) Pairedpulse facilitation and depression at unitary synapses in rat hippocampus: quantal fluctuation affects subsequent release. J Physiol 491:163-176. CrossRef Medline

de Wit J, Sylwestrak E, O'Sullivan ML, Otto S, Tiglio K, Savas JN, Yates JR 3rd, Comoletti D, Taylor P, Ghosh A (2009) LRRTM2 interacts with Neurexin 1 and regulates excitatory synapse formation. Neuron 64:799-806. CrossRef Medline

Etherton M, Földy C, Sharma M, Tabuchi K, Liu X, Shamloo M, Malenka RC, Südhof TC (2011) Autism-linked neuroligin-3 R451C mutation differentially alters hippocampal and cortical synaptic function. Proc Natl Acad Sci U S A 108:13764-13769. CrossRef Medline

Fabrichny IP, Leone P, Sulzenbacher G, Comoletti D, Miller MT, Taylor P, Bourne Y, Marchot P (2007) Structural analysis of the synaptic protein neuroligin and its beta-neurexin complex: determinants for folding and cell adhesion. Neuron 56:979-991. CrossRef Medline

Földy C, Malenka RC, Südhof TC (2013) Autism-associated neuroligin-3 mutations commonly disrupt tonic endocannabinoid signaling. Neuron 78:498-509. CrossRef Medline

Foster KA, Kreitzer AC, Regehr WG (2002) Interaction of postsynaptic receptor saturation with presynaptic mechanisms produces a reliable synapse. Neuron 36:1115-1126. CrossRef Medline

Futai K, Kim MJ, Hashikawa T, Scheiffele P, Sheng M, Hayashi Y (2007) Retrograde modulation of presynaptic release probability through signaling mediated by PSD-95-neuroligin. Nat Neurosci 10:186-195. CrossRef Medline

Gibson JR, Huber KM, Südhof TC (2009) Neuroligin-2 deletion selectively decreases inhibitory synaptic transmission originating from fast-spiking but not from somatostatin-positive interneurons. J Neurosci 29:1388313897. CrossRef Medline

Graf ER, Zhang X, Jin SX, Linhoff MW, Craig AM (2004) Neurexins induce differentiation of GABA and glutamate postsynaptic specializations via neuroligins. Cell 119:1013-1026. CrossRef Medline

Ichtchenko K, Hata Y, Nguyen T, Ullrich B, Missler M, Moomaw C, Südhof TC (1995) Neuroligin 1: a splice site-specific ligand for beta-neurexins. Cell 81:435-443. CrossRef Medline

Ichtchenko K, Nguyen T, Südhof TC (1996) Structures, alternative splicing, and neurexin binding of multiple neuroligins. J Biol Chem 271:26762682. CrossRef Medline

Jedlicka P, Vnencak M, Krueger DD, Jungenitz T, Brose N, Schwarzacher SW (2015) Neuroligin-1 regulates excitatory synaptic transmission, LTP and EPSP-spike coupling in the dentate gyrus in vivo. Brain Struct Funct 220:47-58. CrossRef Medline

Jiang M, Polepalli J, Chen LY, Zhang B, Südhof TC, Malenka RC (2017) Conditional ablation of neuroligin-1 in CA1 pyramidal neurons blocks LTP by a cell-autonomous NMDA receptor-independent mechanism. Mol Psychiatry 22:375-383. CrossRef Medline

Kaeser PS, Deng L, Fan M, Südhof TC (2012) RIM genes differentially contribute to organizing presynaptic release sites. Proc Natl Acad Sci U S A 109:11830-11835. CrossRef Medline

Kim J, Jung SY, Lee YK, Park S, Choi JS, Lee CJ, Kim HS, Choi YB, Scheiffele P, Bailey CH, Kandel ER, Kim JH (2008) Neuroligin-1 is required for normal expression of LTP and associative fear memory in the amygdala of adult animals. Proc Natl Acad Sci U S A 105:9087-9092. CrossRef Medline

Klingauf J, Kavalali ET, Tsien RW (1998) Kinetics and regulation of fast endocytosis at hippocampal synapses. Nature 394:581-585. CrossRef Medline

Ko J, Zhang C, Arac D, Boucard AA, Brunger AT, Südhof TC (2009a) Neuroligin-1 performs neurexin-dependent and neurexin-independent functions in synapse validation. EMBO J 28:3244-3255. CrossRef Medline

Ko J, Fuccillo MV, Malenka RC, Südhof TC (2009b) LRRTM2 functions as a neurexin ligand in promoting excitatory synapse formation. Neuron 64:791-798. CrossRef Medline

Ko J, Soler-Llavina GJ, Fuccillo MV, Malenka RC, Südhof TC (2011) Neuroligins/LRRTMs prevent activity- and $\mathrm{Ca} 2+/$ calmodulin-dependent 
synapse elimination in cultured neurons. J Cell Biol 194:323-334. CrossRef Medline

Krueger DD, Tuffy LP, Papadopoulos T, Brose N (2012) The role of neurexins and neuroligins in the formation, maturation, and function of vertebrate synapses. Curr Opin Neurobiol 22:412-422. CrossRef Medline

Kwon HB, Kozorovitskiy Y, Oh WJ, Peixoto RT, Akhtar N, Saulnier JL, Gu C, Sabatini BL (2012) Neuroligin-1-dependent competition regulates cortical synaptogenesis and synapse number. Nat Neurosci 15:1667-1674. CrossRef Medline

Li J, Ashley J, Budnik V, Bhat MA (2007) Crucial role of Drosophila neurexin in proper active zone apposition to postsynaptic densities, synaptic growth, and synaptic transmission. Neuron 55:741-755. CrossRef Medline

Liang J, Xu W, Hsu YT, Yee AX, Chen L, Südhof TC (2015) Conditional neuroligin-2 knockout in adult medial prefrontal cortex links chronic changes in synaptic inhibition to cognitive impairments. Mol Psychiatry 20:850-859. CrossRef Medline

Malinow R, Tsien RW (1990) Presynaptic enhancement shown by wholecell recordings of long-term potentiation in hippocampal slices. Nature 346:177-180. CrossRef Medline

Manabe T, Nicoll RA (1994) Long-term potentiation: evidence against an increase in transmitter release probability in the CA1 region of the hippocampus. Science 265:1888-1892. CrossRef Medline

Matsuda K, Yuzaki M (2011) Cbln family proteins promote synapse formation by regulating distinct neurexin signaling pathways in various brain regions. Eur J Neurosci 33:1447-1461. CrossRef Medline

Matteoli M, Takei K, Perin MS, Südhof TC, De Camilli P (1992) Exoendocytotic recycling of synaptic vesicles in developing processes of cultured hippocampal neurons. J Cell Biol 117:849-861. CrossRef Medline

Maximov A, Pang ZP, Tervo DG, Südhof TC (2007) Monitoring synaptic transmission in primary neuronal cultures using local extracellular stimulation. J Neurosci Methods 161:75-87. CrossRef Medline

McKinney RA, Capogna M, Dürr R, Gähwiler BH, Thompson SM (1999) Miniature synaptic events maintain dendritic spines via AMPA receptor activation. Nat Neurosci 2:44-49. CrossRef Medline

Missler M, Hammer RE, Südhof TC (1998) Neurexophilin binding to alphaneurexins. A single LNS domain functions as an independently folding ligand-binding unit. J Biol Chem 273:34716-34723. CrossRef Medline

Mondin M, Labrousse V, Hosy E, Heine M, Tessier B, Levet F, Poujol C, Blanchet C, Choquet D, Thoumine O (2011) Neurexin-neuroligin adhesions capture surface-diffusing AMPA receptors through PSD-95 scaffolds. J Neurosci 31:13500-13515. CrossRef Medline

Nguyen T, Südhof TC (1997) Binding properties of neuroligin 1 and neurexin 1beta reveal function as heterophilic cell adhesion molecules. J Biol Chem 272:26032-26039. CrossRef Medline

Owald D, Khorramshahi O, Gupta VK, Banovic D, Depner H, Fouquet W, Wichmann C, Mertel S, Eimer S, Reynolds E, Holt M, Aberle H, Sigrist SJ (2012) Cooperation of Syd-1 with Neurexin synchronizes pre- with postsynaptic assembly. Nat Neurosci 15:1219-1226. CrossRef Medline

Poulopoulos A, Aramuni G, Meyer G, Soykan T, Hoon M, Papadopoulos T, Zhang M, Paarmann I, Fuchs C, Harvey K, Jedlicka P, Schwarzacher SW, Betz H, Harvey RJ, Brose N, Zhang W, Varoqueaux F (2009) Neuroligin 2 drives postsynaptic assembly at perisomatic inhibitory synapses through gephyrin and collybistin. Neuron 63:628-642. CrossRef Medline

Poulopoulos A, Soykan T, Tuffy LP, Hammer M, Varoqueaux F, Brose N (2012) Homodimerization and isoform-specific heterodimerization of neuroligins. Biochem J 446:321-330. CrossRef Medline

Prange O, Wong TP, Gerrow K, Wang YT, El-Husseini A (2004) A balance between excitatory and inhibitory synapses is controlled by PSD-95 and neuroligin. Proc Natl Acad Sci U S A 101:13915-13920. CrossRef Medline Raffaelli G, Saviane C, Mohajerani MH, Pedarzani P, Cherubini E (2004)
BK potassium channels control transmitter release at CA3-CA3 synapses in the rat hippocampus. J Physiol 557:147-157. CrossRef Medline

Rodriguez A, Ehlenberger DB, Dickstein DL, Hof PR, Wearne SL (2008) Automated three-dimensional detection and shape classification of dendritic spines from fluorescence microscopy images. PLoS One 3:e1997. CrossRef Medline

Rosenmund C, Clements JD, Westbrook GL (1993) Nonuniform probability of glutamate release at a hippocampal synapse. Science 262:754-757. CrossRef Medline

Rothwell PE, Fuccillo MV, Maxeiner S, Hayton SJ, Gokce O, Lim BK, Fowler SC, Malenka RC, Südhof TC (2014) Autism-associated neuroligin-3 mutations commonly impair striatal circuits to boost repetitive behaviors. Cell 158:198-212. CrossRef Medline

Shipman SL, Schnell E, Hirai T, Chen BS, Roche KW, Nicoll RA (2011) Functional dependence of neuroligin on a new non-PDZ intracellular domain. Nat Neurosci 14:718-726. CrossRef Medline

Siddiqui TJ, Pancaroglu R, Kang Y, Rooyakkers A, Craig AM (2010) LRRTMs and neuroligins bind neurexins with a differential code to cooperate in glutamate synapse development. J Neurosci 30:7495-7506. CrossRef Medline

Soler-Llavina GJ, Fuccillo MV, Ko J, Südhof TC, Malenka RC (2011) The neurexin ligands, neuroligins and leucine-rich repeat transmembrane proteins, perform convergent and divergent synaptic functions in vivo. Proc Natl Acad Sci U S A 108:16502-16509. CrossRef Medline

Song JY, Ichtchenko K, Südhof TC, Brose N (1999) Neuroligin 1 is a postsynaptic cell-adhesion molecule of excitatory synapses. Proc Natl Acad Sci U S A 96:1100-1105. CrossRef Medline

Südhof TC (2008) Neuroligins and neurexins link synaptic function to $\operatorname{cog}$ nitive disease. Nature 455:903-911. CrossRef Medline

Sun M, Xing G, Yuan L, Gan G, Knight D, With SI, He C, Han J, Zeng X, Fang M, Boulianne GL, Xie W (2011) Neuroligin 2 is required for synapse development and function at the Drosophila neuromuscular junction. J Neurosci 31:687-699. CrossRef Medline

Sutor B, Luhmann HJ (1995) Development of excitatory and inhibitory postsynaptic potentials in the rat neocortex. Perspect Dev Neurobiol 2:409-419. Medline

Tabuchi K, Südhof TC (2002) Structure and evolution of neurexin genes: insight into the mechanism of alternative splicing. Genomics 79:849859. CrossRef Medline

Takács VT, Freund TF, Nyiri G (2013) Neuroligin 2 is expressed in synapses established by cholinergic cells in the mouse brain. PLoS One 8:e72450. CrossRef Medline

Varoqueaux F, Aramuni G, Rawson RL, Mohrmann R, Missler M, Gottmann K, Zhang W, Südhof TC, Brose N (2006) Neuroligins determine synapse maturation and function. Neuron 51:741-754. CrossRef Medline

Varoqueaux F, Jamain S, Brose N (2004) Neuroligin 2 is exclusively localized to inhibitory synapses. Eur J Cell Biol 83:449-456. CrossRef Medline

Wadiche JI, Jahr CE (2001) Multivesicular release at climbing fiber-Purkinje cell synapses. Neuron 32:301-313. CrossRef Medline

Wong AY, Graham BP, Billups B, Forsythe ID (2003) Distinguishing between presynaptic and postsynaptic mechanisms of short-term depression during action potential trains. J Neurosci 23:4868-4877. Medline

Xing G, Gan G, Chen D, Sun M, Yi J, Lv H, Han J, Xie W (2014) Drosophila neuroligin3 regulates neuromuscular junction development and synaptic differentiation. J Biol Chem 289:31867-31877. CrossRef Medline

Zhang B, Chen LY, Liu X, Maxeiner S, Lee SJ, Gokce O, Südhof TC (2015) Neuroligins sculpt cerebellar Purkinje-cell circuits by differential control of distinct classes of synapses. Neuron 87:781-796. CrossRef Medline 